L. Costa and R. M. Miró-Roig

Nagoya Math. J.

Vol. 186 (2007), 119-155

\title{
m-BLOCKS COLLECTIONS AND CASTELNUOVO-MUMFORD REGULARITY IN MULTIPROJECTIVE SPACES
}

\author{
L. COSTA* AND R. M. MIRÓ-ROIG**
}

\begin{abstract}
The main goal of the paper is to generalize Castelnuovo-Mumford regularity for coherent sheaves on projective spaces to coherent sheaves on $n$ dimensional smooth projective varieties $X$ with an $n$-block collection $\mathcal{B}$ which generates the bounded derived category $\mathcal{D}^{b}\left(\mathcal{O}_{X}-\bmod \right)$. To this end, we use the theory of $n$-blocks and Beilinson type spectral sequence to define the notion of regularity of a coherent sheaf $F$ on $X$ with respect to the $n$-block collection $\mathcal{B}$. We show that the basic formal properties of the Castelnuovo-Mumford regularity of coherent sheaves over projective spaces continue to hold in this new setting and we compare our definition of regularity with previous ones. In particular, we show that in case of coherent sheaves on $\mathbb{P}^{n}$ and for the $n$ block collection $\mathcal{B}=\left(\mathcal{O}_{\mathbb{P}^{n}}, \mathcal{O}_{\mathbb{P}^{n}}(1), \ldots, \mathcal{O}_{\mathbb{P}^{n}}(n)\right)$ on $\mathbb{P}^{n}$ Castelnuovo-Mumford regularity and our new definition of regularity coincide. Finally, we carefully study the regularity of coherent sheaves on a multiprojective space $\mathbb{P}^{n_{1}} \times \cdots \times$ $\mathbb{P}^{n_{r}}$ with respect to a suitable $n_{1}+\cdots+n_{r}$-block collection and we compare it with the multigraded variant of the Castelnuovo-Mumford regularity given by Hoffman and Wang in [14].
\end{abstract}

\section{§1. Introduction}

In Chapter 14 of [19] D. Mumford introduced the concept of regularity for a coherent sheaf $F$ on a projective space $\mathbb{P}^{n}$ to bound the family of all projective subschemes having fixed Hilbert polynomial. Since then Castelnuovo-Mumford regularity has become a fundamental invariant in commutative algebra and algebraic geometry. It measures the complexity of a module or a sheaf; more precisely the regularity of a module bounds the largest degree of the minimal generators and the degree of syzygies and the regularity of a sheaf estimates the smallest twist for which the sheaf is

Received July 27, 2005.

Revised March 13, 2006.

1991 Mathematics Subject Classification: 14F05.

*Partially supported by MTM2004-00666.

** Partially supported by MTM2004-00666. 
globally generated.

Let $X$ be a smooth projective variety of dimension $n$ and let $\mathcal{B}=$ $\left(\mathcal{E}_{0}, \ldots, \mathcal{E}_{n}\right)$ be an $n$-block collection of objects of $\mathcal{D}^{b}\left(\mathcal{O}_{X}-\bmod \right)$ which generates the bounded derived category $\mathcal{D}^{b}\left(\mathcal{O}_{X}-\bmod \right)$. The goal of this paper is to introduce the notion of regularity of a coherent sheaf on $X$ with respect to $\mathcal{B}$ as a generalization of the notion of Castelnuovo-Mumford regularity of coherent sheaves on projective spaces. To introduce this new notion of regularity and to state the basic formal properties, we use helix theory, $m$-blocks collections and Beilinson type spectral sequences.

We want to stress that Castelnuovo-Mumford regularity as well as the notion of regularity developed for Grassmannians in [4] and multiprojective spaces in [14] fall under the umbrella of $\mathcal{B}$-regularity. Moreover, in this new setting, we are able to prove analogs of some of the classical results on $m$-regularity for coherent sheaves on projective spaces.

Next we outline the structure of the paper. In Section 2, we briefly recall the notions and properties of full strongly exceptional collections of sheaves on a smooth projective variety needed later. The notion of $m$-block as well as the concept of mutations of blocks are presented in Section 3. Sections 4 and 5 are the heart of the paper. In Section 4, we first introduce the notion of helix of blocks associated to an $m$-block collection of objects of $\mathcal{D}^{b}\left(\mathcal{O}_{X}-\right.$ mod $)$ as a natural generalization of the notion of helix associated to an exceptional collection of objects of $\mathcal{D}^{b}\left(\mathcal{O}_{X}-\bmod \right)$. Then, using Beilinson type spectral sequences, we give the promised definition of regularity of a coherent sheaf $F$ on $X$ with respect to an $n$-block collection $\mathcal{B}$ which generates $\mathcal{D}^{b}\left(\mathcal{O}_{X}-\bmod \right)$, we prove that the Castelnuovo-Mumford regularity of a coherent sheaf $F$ on $\mathbb{P}^{n}$ coincides with the regularity of $F$ with respect to a suitable $n$-block collection on $\mathbb{P}^{n}$ and we show that the main formal properties of the Castelnuovo-Mumford regularity carry over to the new setting. In Section 5, we restrict our attention to multiprojective spaces $X=\mathbb{P}^{n_{1}} \times \cdots \times \mathbb{P}^{n_{r}}$ and we analyze the relationship between our definition of regularity and the multigraded variant of the Castelnuovo-Mumford regularity introduced by Hoffman and Wang in [14]. Finally, in Section 6, we collect some questions which naturally arise from this paper.

Notation. Throughout this paper $X$ will be a smooth projective variety defined over the complex numbers $\mathbb{C}$ (most of the results are true for varieties over an algebraically closed field) and we denote by $\mathcal{D}=\mathcal{D}^{b}\left(\mathcal{O}_{X}-\right.$ mod $)$ 
the derived category of bounded complexes of coherent sheaves of $\mathcal{O}_{X^{-}}$ modules. Notice that $\mathcal{D}$ is an abelian linear triangulated category. We identify, as usual, any coherent sheaf $F$ on $X$ to the object $(0 \rightarrow F \rightarrow 0) \in \mathcal{D}$ concentrated in degree zero and we will not distinguish between a vector bundle and its locally free sheaf of sections.

\section{$\S 2$. Basic facts on exceptional collections}

As we pointed out in the introduction, in this section we gather the basic definitions and properties on full strongly exceptional collections of sheaves on a smooth projective variety needed in the sequel. For general facts on triangulated categories see [20].

Definition 2.1. Let $X$ be a smooth projective variety.

(i) An object $F \in \mathcal{D}$ is exceptional if $\operatorname{Hom}_{\mathcal{D}}^{\bullet}(F, F)$ is a 1-dimensional algebra generated by the identity.

(ii) An ordered collection $\left(F_{0}, F_{1}, \ldots, F_{m}\right)$ of objects of $\mathcal{D}$ is an exceptional collection if each object $F_{i}$ is exceptional and $\operatorname{Ext}_{\mathcal{D}}^{\bullet}\left(F_{k}, F_{j}\right)=0$ for $j<k$.

(iii) An exceptional collection $\left(F_{0}, F_{1}, \ldots, F_{m}\right)$ of objects of $\mathcal{D}$ is a strongly exceptional collection if in addition $\operatorname{Ext}_{\mathcal{D}}^{i}\left(F_{j}, F_{k}\right)=0$ for $i \neq 0$ and $j \leq k$.

(iv) An ordered collection of objects of $\mathcal{D},\left(F_{0}, F_{1}, \ldots, F_{m}\right)$, is a full (strongly) exceptional collection if it is a (strongly) exceptional collection and $F_{0}, F_{1}, \ldots, F_{m}$ generate the bounded derived category $\mathcal{D}$.

Remark 2.2. The existence of a full strongly exceptional collection $\left(F_{0}, F_{1}, \ldots, F_{m}\right)$ of coherent sheaves on a smooth projective variety $X$ imposes rather a strong restriction on $X$, namely that the Grothendieck group $K_{0}(X)=K_{0}\left(\mathcal{O}_{X}\right.$-mod $)$ is isomorphic to $\mathbb{Z}^{m+1}$.

Let us illustrate the above definition with precise examples:

EXAMPLE 2.3. (1) $\left(\mathcal{O}_{\mathbb{P} r}, \mathcal{O}_{\mathbb{P}^{r}}(1), \ldots, \mathcal{O}_{\mathbb{P}}(r)\right)$ is a full strongly exceptional collection of coherent sheaves on $\mathbb{P}^{r}$ and $\left(\Omega_{\mathbb{P}^{r}}^{r}(r), \Omega_{\mathbb{P}^{r}}^{r-1}(r-1), \ldots\right.$, $\left.\Omega_{\mathbb{P}^{r}}^{1}(1), \mathcal{O}_{\mathbb{P}^{r}}\right)$ is also a full strongly exceptional collection of coherent sheaves on $\mathbb{P}^{r}$.

(2) Let $\mathbb{F}_{n}=\mathbb{P}\left(\mathcal{O}_{\mathbb{P}^{1}} \oplus \mathcal{O}_{\mathbb{P}^{1}}(n)\right), n \geq 0$, be a Hirzebruch surface. Denote by $\xi$ (resp. $F$ ) the class of the tautological line bundle (resp. the class of a 
fiber of the natural projection $\left.p: \mathbb{F}_{n} \rightarrow \mathbb{P}^{1}\right)$. Then, $(\mathcal{O}, \mathcal{O}(F), \mathcal{O}(\xi), \mathcal{O}(F+$ $\xi))$ is a full strongly exceptional collection of coherent sheaves on $\mathbb{F}_{n}$.

(3) $\left(\mathcal{O}_{\mathbb{P}^{n}}(-n) \bigotimes \mathcal{O}_{\mathbb{P}^{m}}(-m), \mathcal{O}_{\mathbb{P}^{n}}(-n+1) \bigotimes \mathcal{O}_{\mathbb{P}^{m}}(-m), \ldots, \mathcal{O}_{\mathbb{P}^{n}} \otimes \mathcal{O}_{\mathbb{P}^{m}}(-m)\right.$, $\left.\ldots, \mathcal{O}_{\mathbb{P}^{n}}(-n) \otimes \mathcal{O}_{\mathbb{P}^{m}}, \mathcal{O}_{\mathbb{P}^{n}}(-n+1) \otimes \mathcal{O}_{\mathbb{P}^{m}}, \ldots, \mathcal{O}_{\mathbb{P}^{n}} \otimes \mathcal{O}_{\mathbb{P}^{m}}\right)$ is a full strongly exceptional collection of locally free sheaves on $\mathbb{P}^{n} \times \mathbb{P}^{m}$ (see also [5]; Proposition 4.16).

(4) Let $\pi: \widetilde{\mathbb{P}}^{2}(l) \rightarrow \mathbb{P}^{2}$ be the blow up of $\mathbb{P}^{2}$ at $l$ points and let $E_{1}=$ $\pi^{-1}\left(p_{1}\right), \ldots, E_{l}=\pi^{-1}\left(p_{l}\right)$ be the exceptional divisors. Then, the collection of line bundles on $\widetilde{\mathbb{P}}^{2}(l)$

$$
\left(\mathcal{O}, \mathcal{O}\left(E_{1}\right), \mathcal{O}\left(E_{2}\right), \ldots, \mathcal{O}\left(E_{l}\right), \mathcal{O}(H), \mathcal{O}(2 H)\right)
$$

is a full strongly exceptional collection of coherent sheaves on $\widetilde{\mathbb{P}}^{2}(l)$.

(5) Let $X=G r(k, n)$ be the Grassmannian of $k$-dimensional subspaces of the $n$-dimensional vector space. We have the canonical exact sequence

$$
0 \longrightarrow \mathcal{S} \longrightarrow \mathcal{O}_{X}^{n} \longrightarrow \mathcal{Q} \longrightarrow 0
$$

where $\mathcal{S}$ denotes the tautological $k$-dimensional bundle and $\mathcal{Q}$ the quotient bundle. In the sequel, $\Sigma^{\alpha} \mathcal{S}$ denotes the space of the irreducible representations of the group $G L(\mathcal{S})$ with highest weight $\alpha=\left(\alpha_{1}, \ldots, \alpha_{s}\right)$ and $|\alpha|=\sum_{i=1}^{s} \alpha_{i}$ (see [10] for general facts on Weyl modules). Denote by $A(k, n)$ the set of locally free sheaves $\Sigma^{\alpha} \mathcal{S}$ on $\operatorname{Gr}(k, n)$ where $\alpha$ runs over Young diagrams fitting inside a $k \times(n-k)$ rectangle. Notice that for any $\Sigma^{\alpha} \mathcal{S} \in A(k, n), 0 \leq|\alpha| \leq k(n-k)$. Set $\rho(k, n):=\sharp A(k, n)$. By [15]; Proposition 2.2 (a), for any $\Sigma^{\alpha} \mathcal{S}, \Sigma^{\beta} \mathcal{S} \in A(k, n), \operatorname{Ext}^{i}\left(\Sigma^{\alpha} \mathcal{S}, \Sigma^{\beta} \mathcal{S}\right) \neq 0$ only if $i=0$ and by [16] (3.5), $\operatorname{Hom}\left(\Sigma^{\alpha} \mathcal{S}, \Sigma^{\beta} \mathcal{S}\right) \neq 0$ only if $\alpha_{i} \geq \beta_{i}$ for all $i$. Denote by $\mathcal{E}_{r}$ the set of bundles $\Sigma^{\alpha} \mathcal{S} \in A(k, n)$ with $|\alpha|=k(n-k)-r$. Let $\sigma$ be the ordered collection of locally free sheaves on $X$ constructed in the following way. Going from the left to the right, first put all the $\Sigma^{\alpha} \mathcal{S} \in \mathcal{E}_{0}$, i.e. all the $\Sigma^{\alpha} \mathcal{S} \in A(k, n)$ with $|\alpha|=k(n-k)$. The $i$-th time put all the $\Sigma^{\alpha} \mathcal{S} \in \mathcal{E}_{i}$, i.e. all the $\Sigma^{\alpha} \mathcal{S} \in A(k, n)$ with $|\alpha|=k(n-k)-i$ and so on until $i=k(n-k)$. By construction $\sigma$ is a strongly exceptional collection and by [15] Proposition 1.4, it is full. So, $A(k, n)$ can be totally ordered in such a way that we obtain a full strongly exceptional collection $\left(E_{1}, \ldots, E_{\rho(k, n)}\right)$ of locally free sheaves on $X$.

(6) Let $Q_{n} \subset \mathbb{P}^{n+1}, n>2$, be a hyperquadric surface. By [16]; Proposition 4.9 , if $n$ is even and $\Sigma_{1}, \Sigma_{2}$ are the Spinor bundles on $Q_{n}$, then

$$
\left(\Sigma_{1}(-n), \Sigma_{2}(-n), \mathcal{O}_{Q_{n}}(-n+1), \ldots, \mathcal{O}_{Q_{n}}(-1), \mathcal{O}_{Q_{n}}\right)
$$


is a full strongly exceptional collection of locally free sheaves on $Q_{n}$; and if $n$ is odd and $\Sigma$ is the Spinor bundle on $Q_{n}$, then

$$
\left(\Sigma(-n), \mathcal{O}_{Q_{n}}(-n+1), \ldots, \mathcal{O}_{Q_{n}}(-1), \mathcal{O}_{Q_{n}}\right)
$$

is a full strongly exceptional collection of locally free sheaves on $Q_{n}$.

The importance of the existence of full strongly exceptional collections relies on the fact that each full strongly exceptional collection $\left(F_{0}, F_{1}, \ldots\right.$, $F_{m}$ ) of coherent sheaves on a smooth projective variety $X$ determines a tilting sheaf $\mathcal{T}=\bigoplus_{i=0}^{m} F_{i}$ and hence functors $\operatorname{RHom}_{X}(\mathcal{T},-): D^{b}\left(\mathcal{O}_{X}-\bmod \right) \rightarrow$ $D^{b}(A)$ and $-\otimes_{A}^{\mathbf{L}} \mathcal{T}: D^{b}(A) \rightarrow D^{b}\left(\mathcal{O}_{X}-\bmod \right)$ which define mutually inverse equivalences between the bounded derived categories of coherent sheaves on $X$ and the bounded derived category of finitely generated right $A=$ $\operatorname{Hom}_{X}(\mathcal{T}, \mathcal{T})$-modules, respectively.

Definition 2.4. Let $X$ be a smooth projective variety and let $(A, B)$ be an exceptional pair of objects of $\mathcal{D}$. We define objects $L_{A} B$ and $R_{B} A$ with the aid of the following distinguished triangles in the category $\mathcal{D}$ :

$$
\begin{gathered}
L_{A} B \longrightarrow \operatorname{Hom}_{\mathcal{D}}^{\bullet}(A, B) \otimes A \longrightarrow B \longrightarrow L_{A} B[1] \\
R_{B} A[-1] \longrightarrow A \longrightarrow \operatorname{Hom}_{\mathcal{D}}^{\times \bullet}(A, B) \otimes B \longrightarrow R_{B} A
\end{gathered}
$$

A left mutation of an exceptional pair $\sigma=(A, B)$ is the pair

$$
L_{A} \sigma=\left(L_{A} B, A\right)=(L B, A)
$$

and a right mutation of an exceptional pair $\sigma=(A, B)$ is the pair

$$
R_{A} \sigma=\left(B, R_{B} A\right)=(B, R A) .
$$

Lower indices will be omitted whenever this does not cause confusion.

Definition 2.5. Let $X$ be a smooth projective variety and let $\sigma=$ $\left(E_{0}, \ldots, E_{m}\right)$ be an exceptional collection of objects of $\mathcal{D}$. A left mutation (resp. right mutation) of $\sigma$ is defined as a mutation of a pair of adjacent objects in this collection, i.e. for any $1 \leq i \leq m$ a left mutation $L_{i}$ replaces the $i$-th pair of consequent elements $\left(E_{i-1}, E_{i}\right)$ by its left mutation $\left(L_{E_{i-1}} E_{i}, E_{i-1}\right)$ and a right mutation $R_{i}$ replaces the same pair of consequent elements $\left(E_{i-1}, E_{i}\right)$ by its right mutation $\left(E_{i}, R_{E_{i}} E_{i-1}\right)$ :

$$
\begin{gathered}
L_{i} \sigma=L_{E_{i-1}} \sigma=\left(E_{0}, \ldots, L_{E_{i-1}} E_{i}, E_{i-1}, \ldots, E_{m}\right) \\
R_{i} \sigma=R_{E_{i-1}} \sigma=\left(E_{0}, \ldots, E_{i}, R_{E_{i}} E_{i-1}, \ldots, E_{m}\right) .
\end{gathered}
$$


Notation 2.6. Let $X$ be a smooth projective variety and let $\sigma=$ $\left(F_{0}, \ldots, F_{m}\right)$ be an exceptional collection of objects of $\mathcal{D}$. It is convenient to agree that for any $0 \leq i, j \leq m$ and $i+j \leq m$,

$$
\begin{gathered}
R^{(j)} F_{i}=R^{(j-1)} R F_{i}=R_{F_{i+j}} \cdots R_{F_{i+2}} R_{F_{i+1}} F_{i}=: R_{F_{i+j} \cdots F_{i+2} F_{i+1}} F_{i} \\
R_{F_{i-1}}^{(0)} \sigma=\sigma \quad R_{F_{i-1}}^{(j)} \sigma=R_{R F_{i-1}}^{(j-1)}\left(R_{F_{i-1}} \sigma\right)
\end{gathered}
$$

and similar notation for compositions of left mutations.

Remark 2.7. (1) If $X$ is a smooth projective variety and $\sigma=\left(F_{0}, \ldots\right.$, $\left.F_{m}\right)$ is an exceptional collection of objects of $\mathcal{D}$, then any mutation of $\sigma$ is an exceptional collection. Moreover, if $\sigma$ generates the category $\mathcal{D}$, then the mutated collection also generates $\mathcal{D}$.

(2) In general, a mutation of a strongly exceptional collection is not a strongly exceptional collection. In fact, take $X=\mathbb{P}^{1} \times \mathbb{P}^{1}$ and consider the full strongly exceptional collection $\sigma=\left(\mathcal{O}_{X}, \mathcal{O}_{X}(1,0), \mathcal{O}_{X}(0,1), \mathcal{O}_{X}(1,1)\right)$ of line bundles on $X$. It is not difficult to check that the mutated collection

$$
\begin{gathered}
\left(\mathcal{O}_{X}, \mathcal{O}_{X}(1,0), L_{\mathcal{O}_{X}(0,1)} \mathcal{O}_{X}(1,1), \mathcal{O}_{X}(0,1)\right) \\
=\left(\mathcal{O}_{X}, \mathcal{O}_{X}(1,0), \mathcal{O}_{X}(-1,1), \mathcal{O}_{X}(0,1)\right)
\end{gathered}
$$

is no more a strongly exceptional collection of line bundles on $X$.

Let $X$ be a smooth projective variety of dimension $n$. It is well known that if full strongly exceptional collections of coherent sheaves on $X$ exist then all of them have the same length and it is equal to the rank of $K_{0}(X)$. Even more, this length is bounded below by $n+1$ because for any smooth projective variety $X$ of dimension $n$ we have $\operatorname{rank}\left(K_{0}(X)\right) \geq n+1$. In [6]; we give the following definition (see also [3] and [13]):

DEFinition 2.8. Let $X$ be a smooth projective variety of dimension $n$. We say that an ordered collection of coherent sheaves $\sigma=\left(E_{0}, \ldots, E_{n}\right)$ is a geometric collection if it is a full exceptional collection of coherent sheaves on $X$ of minimal length, $n+1$, i.e. of length one greater than the dimension of $X$.

By [2]; Assertion 9.2, Theorem 9.3 and Corollary 9.4, geometric collections are automatically strongly exceptional collections of coherent sheaves and the strongly exceptionality is preserved under mutations. 
EXAMPLE 2.9. (1) The collection $\sigma=\left(\mathcal{O}_{\mathbb{P}^{r}}(-r), \mathcal{O}_{\mathbb{P}^{r}}(-r+1), \mathcal{O}_{\mathbb{P}^{r}}(-r+\right.$ $\left.2), \ldots, \mathcal{O}_{\mathbb{P}^{r}}\right)$ of line bundles on $\mathbb{P}^{r}$ is a geometric collection of coherent sheaves.

(2) If $n$ is odd and $Q_{n} \subset \mathbb{P}^{n+1}$ is a quadric hypersurface, the collection of locally free sheaves

$$
\left(\Sigma(-n), \mathcal{O}_{Q_{n}}(-n+1), \ldots, \mathcal{O}_{Q_{n}}(-1), \mathcal{O}_{Q_{n}}\right)
$$

being $\Sigma$ the Spinor bundle on $Q_{n}$ is a geometric collection of locally free sheaves on $Q_{n}$.

(3) If $n$ is even and $Q_{n} \subset \mathbb{P}^{n+1}$ is a quadric hypersurface, the collection of locally free sheaves

$$
\left(\Sigma_{1}(-n), \Sigma_{2}(-n), \mathcal{O}_{Q_{n}}(-n+1), \ldots, \mathcal{O}_{Q_{n}}(-1), \mathcal{O}_{Q_{n}}\right)
$$

being $\Sigma_{1}$ and $\Sigma_{2}$ the Spinor bundles on $Q_{n}$, is a full strongly exceptional collection of locally free sheaves on $Q_{n}$. Since all full strongly exceptional collections of coherent sheaves on $Q_{n}$ have length $n+2$, we conclude that there are no geometric collections of coherent sheaves on $Q_{n}$ for even $n$.

(4) It follows from Example 2.3 (5) that there are no geometric collections of coherent sheaves on $\operatorname{Gr}(k, n)$ if $k \neq n-1$.

(5) Any smooth Fano threefold $X$ with $\operatorname{Pic}(X) \cong \mathbb{Z}$ and trivial intermediate Jacobian has a geometric collection (see [6]; Proposition 3.6).

In [6], the authors extend the notion of Castelnuovo-Mumford regularity for coherent sheaves on projective spaces to coherent sheaves on smooth projective varieties with a geometric collection. So we are led to consider the following problem:

Problem 2.10. To characterize the smooth projective varieties which have a geometric collection.

To our knowledge Problem 2.10 is far of being solved (see [6] for more information). Moreover, we want to stress that the existence of a geometric collection on an $n$-dimensional smooth variety $X$ imposes a strong restriction on $X$; e.g. $X$ has to be a Fano variety ([3]; Theorem 3.4) and the Grothendieck group $K_{0}(X)$ has to be a $\mathbb{Z}$-free module of rank $n+1$. So, it is convenient to generalize the notion of geometric collection in order to be able to extend the concept of Castelnuovo-Mumford regularity for coherent 
sheaves on projective spaces to coherent sheaves on smooth projective varieties as Grassmannians, even-dimensional hyperquadrics, multiprojective spaces, etc., which do not have geometric collections. This will be achieved allowing exceptional collections $\sigma=\left(F_{0}, \ldots, F_{m}\right)$ of arbitrary length but packing the objects $F_{i} \in \mathcal{D}$ in suitable subcollections called blocks.

\section{$\S 3 . \quad m$-blocks and mutations}

The notion of block was introduced by Karpov and Nogin in [17]. We start this section recalling its definition and properties (see also [13]).

Definition 3.1. (i) An exceptional collection $\left(F_{0}, F_{1}, \ldots, F_{m}\right)$ of objects of $\mathcal{D}$ is a block if $\operatorname{Ext}_{\mathcal{D}}^{i}\left(F_{j}, F_{k}\right)=0$ for any $i$ and $j \neq k$.

(ii) An m-block collection of type $\left(\alpha_{0}, \alpha_{1}, \ldots, \alpha_{m}\right)$ of objects of $\mathcal{D}$ is an exceptional collection

$$
\mathcal{B}=\left(\mathcal{E}_{0}, \mathcal{E}_{1}, \ldots, \mathcal{E}_{m}\right)=\left(E_{1}^{0}, \ldots, E_{\alpha_{0}}^{0}, E_{1}^{1}, \ldots, E_{\alpha_{1}}^{1}, \ldots, E_{1}^{m}, \ldots, E_{\alpha_{m}}^{m}\right)
$$

such that all the subcollections $\mathcal{E}_{j}=\left(E_{1}^{j}, E_{2}^{j}, \ldots, E_{\alpha_{j}}^{j}\right)$ are blocks.

Note that an exceptional collection $\left(E_{0}, E_{1}, \ldots, E_{m}\right)$ is an $m$-block of type $(1,1, \ldots, 1)$.

EXAMPLE 3.2. (1) $\left(\mathcal{O}_{\mathbb{P} r}(-r), \mathcal{O}_{\mathbb{P} r}(-r+1), \mathcal{O}_{\mathbb{P}^{r}}(-r+2), \ldots, \mathcal{O}_{\mathbb{P}^{r}}\right)$ is an $r$-block of type $(1,1, \ldots, 1)$.

(2) Let $X=\operatorname{Gr}(k, n)$ be the Grassmannian of $k$-dimensional subspaces of the $n$-dimensional vector space, $k>1$. In Example 2.3 (4), we have seen that $A(k, n)$ can be totally ordered in such a way that we obtain a full strongly exceptional collection

$$
\sigma=\left(E_{1}, \ldots, E_{\rho(k, n)}\right)
$$

of locally free sheaves on $X$. Moreover, packing in the same block $\mathcal{E}_{r}$ the bundles $\Sigma^{\alpha} \mathcal{S} \in \sigma$ with $|\alpha|=k(n-k)-r$ we obtain

$$
\sigma=\left(E_{1}, \ldots, E_{\rho(k, n)}\right)=\left(\mathcal{E}_{0}, \ldots, \mathcal{E}_{k(n-k)}\right)
$$

a $k(n-k)$-block collection of locally free sheaves on $X$ (see Example $2.3(4)$ for details).

(3) Let $Q_{n} \subset \mathbb{P}^{n+1}, n \geq 2$, be a hyperquadric variety. According to Example $2.3(5)$, if $n$ is even and $\Sigma_{1}, \Sigma_{2}$ are the Spinor bundles on $Q_{n}$, then

$$
\left(\Sigma_{1}(-n), \Sigma_{2}(-n), \mathcal{O}_{Q_{n}}(-n+1), \ldots, \mathcal{O}_{Q_{n}}(-1), \mathcal{O}_{Q_{n}}\right)
$$


is a full strongly exceptional collection of locally free sheaves on $Q_{n}$; and if $n$ is odd and $\Sigma$ is the Spinor bundle on $Q_{n}$, then

$$
\left(\Sigma(-n), \mathcal{O}_{Q_{n}}(-n+1), \ldots, \mathcal{O}_{Q_{n}}(-1), \mathcal{O}_{Q_{n}}\right)
$$

is a full strongly exceptional collection of locally free sheaves on $Q_{n}$. Since $\operatorname{Ext}^{i}\left(\Sigma_{1}, \Sigma_{2}\right)=0$ for any $i \geq 0$, we get that $\left(\mathcal{E}_{0}, \mathcal{E}_{1}, \ldots, \mathcal{E}_{n}\right)$ where

$$
\mathcal{E}_{j}=\mathcal{O}_{Q_{n}}(-n+j) \quad \text { for } 1 \leq j \leq n, \quad \mathcal{E}_{0}= \begin{cases}\left(\Sigma_{1}(-n), \Sigma_{2}(-n)\right) & \text { if } n \text { even } \\ (\Sigma(-n)) & \text { if } n \text { odd }\end{cases}
$$

is an $n$-block collection of locally free sheaves on $Q_{n}$ for all $n$.

(4) Let $X=\mathbb{P}^{n_{1}} \times \cdots \times \mathbb{P}^{n_{s}}$ be a multiprojective space of dimension $d=n_{1}+\cdots+n_{s}$. For any $1 \leq i \leq s$, denote by $p_{i}: X \rightarrow \mathbb{P}^{n_{i}}$ the natural projection and write

$$
\mathcal{O}_{X}\left(a_{1}, a_{2}, \ldots, a_{s}\right):=p_{1}^{*} \mathcal{O}_{\mathbb{P}^{n_{1}}}\left(a_{1}\right) \otimes p_{2}^{*} \mathcal{O}_{\mathbb{P}^{n_{2}}}\left(a_{2}\right) \otimes \cdots \otimes p_{s}^{*} \mathcal{O}_{\mathbb{P}^{n_{s}}}\left(a_{s}\right) .
$$

For any $0 \leq j \leq d$, denote by $\mathcal{E}_{j}$ the collection of all line bundles on $X$

$$
\mathcal{O}_{X}\left(a_{1}^{j}, a_{2}^{j}, \ldots, a_{s}^{j}\right)
$$

with $-n_{i} \leq a_{i}^{j} \leq 0$ and $\sum_{i=1}^{s} a_{i}^{j}=j-d$. Using the Künneth formula for locally free sheaves on algebraic varieties, we prove that each $\mathcal{E}_{j}$ is a block and that

$$
\mathcal{B}=\left(\mathcal{E}_{0}, \mathcal{E}_{1}, \ldots, \mathcal{E}_{d}\right)
$$

is a $d$-block collection of line bundles on $X$.

We will now introduce the notion of mutation of block collections.

DeFinition 3.3. Let $X$ be a smooth projective variety and consider a 1-block collection $(\mathcal{E}, \mathcal{F})=\left(E_{1}, \ldots, E_{n}, F_{1}, \ldots, F_{m}\right)$ of objects of $\mathcal{D}$. A left mutation of $F_{j}$ by $\mathcal{E}$ is the object defined by (see Notation 2.6)

$$
L_{\mathcal{E}} F_{j}:=L_{E_{1} E_{2} \cdots E_{n}} F_{j}
$$

and a right mutation of $E_{j}$ by $\mathcal{F}$ is the object defined by

$$
R_{\mathcal{F}} E_{j}:=R_{F_{m} F_{m-1} \cdots F_{1}} E_{j} .
$$

A left mutation of $(\mathcal{E}, \mathcal{F})$ is the pair $\left(L_{\mathcal{E}} \mathcal{F}, \mathcal{E}\right)$ where

$$
L_{\mathcal{E}} \mathcal{F}:=\left(L_{\mathcal{E}} F_{1}, L_{\mathcal{E}} F_{2}, \ldots, L_{\mathcal{E}} F_{m}\right)
$$

and a right mutation of $(\mathcal{E}, \mathcal{F})$ is the pair $\left(\mathcal{F}, R_{\mathcal{F}} \mathcal{E}\right)$ where

$$
R_{\mathcal{F}} \mathcal{E}:=\left(R_{\mathcal{F}} E_{1}, R_{\mathcal{F}} E_{2}, \ldots, R_{\mathcal{F}} E_{n}\right) .
$$


Note that by [11] $(2.2), L_{\mathcal{E}} \mathcal{F}$ and $R_{\mathcal{F}} \mathcal{E}$ are blocks and the pairs $\left(L_{\mathcal{E}} \mathcal{F}, \mathcal{E}\right)$ and $\left(\mathcal{F}, R_{\mathcal{F}} \mathcal{E}\right)$ are 1 -block collections.

Remark 3.4. It follows from the proof of [17]; Proposition 2.2 and Proposition 2.3 that given a 1-block collection $(\mathcal{E}, \mathcal{F})=\left(E_{1}, \ldots, E_{n}, F_{1}, \ldots\right.$, $\left.F_{m}\right)$, the objects $L_{\mathcal{E}} F_{j}$ and $R_{\mathcal{F}} E_{j}$ can be defined with the aid of the following distinguished triangles in the category $\mathcal{D}$

$$
\begin{aligned}
& L_{\mathcal{E}} F_{j} \longrightarrow \bigoplus_{i=1}^{n} \operatorname{Hom}_{\mathcal{D}}^{\bullet}\left(E_{i}, F_{j}\right) \otimes E_{i} \longrightarrow F_{j} \longrightarrow L_{\mathcal{E}} F_{j}[1] \\
& R_{\mathcal{F}} E_{j}[-1] \longrightarrow E_{j} \longrightarrow \bigoplus_{i=1}^{m} \operatorname{Hom}_{\mathcal{D}}^{\times \bullet}\left(E_{j}, F_{i}\right) \otimes F_{i} \longrightarrow R_{\mathcal{F}} E_{j} .
\end{aligned}
$$

Applying $\operatorname{Hom}_{\mathcal{D}}^{\bullet}\left(E_{i}, *\right)$ to the triangle (3.1) we get the orthogonality relation

$$
\operatorname{Hom}_{\mathcal{D}}^{\bullet}\left(E_{i}, L_{\mathcal{E}} F_{j}\right)=0 \quad \text { for all } 1 \leq i \leq n
$$

i.e., $L_{\mathcal{E}} F_{j} \in[\mathcal{E}]^{\perp}:=\left\{F \in \mathcal{D} \mid \operatorname{Hom}_{\mathcal{D}}^{\bullet}(E, F)=0\right.$ for all $\left.E \in[\mathcal{E}]\right\}$, where we denote by $[\mathcal{E}]$ the full triangulated subcategory of $\mathcal{D}$ generated by $E_{1}, \ldots, E_{n}$.

Similarly, $\operatorname{Hom}_{\mathcal{D}}^{\bullet}\left(*, F_{j}\right)$ applied to the triangle (3.2) gives the orthogonality relation

$$
\operatorname{Hom}_{\mathcal{D}}^{\bullet}\left(R_{\mathcal{F}} E_{i}, F_{j}\right)=0 \quad \text { for all } 1 \leq j \leq m
$$

i.e., $R_{\mathcal{F}} E_{i} \in{ }^{\perp}[\mathcal{F}]:=\left\{E \in \mathcal{D} \mid \operatorname{Hom}_{\mathcal{D}}^{\bullet}(E, F)=0\right.$ for all $\left.F \in[\mathcal{F}]\right\}$.

Notation 3.5. It is convenient to agree that

$$
\begin{aligned}
R^{(j)} \mathcal{E}_{i} & =R^{(j-1)} R \mathcal{E}_{i}=R_{\mathcal{E}_{i+j}} \cdots R_{\mathcal{E}_{i+2}} R_{\mathcal{E}_{i+1}} \mathcal{E}_{i}=: R_{\mathcal{E}_{i+j} \cdots \mathcal{E}_{i+2} \mathcal{E}_{i+1}} \mathcal{E}_{i} \\
L^{(j)} \mathcal{E}_{i} & =L^{(j-1)} L \mathcal{E}_{i}=L_{\mathcal{E}_{i-j}} \cdots L_{\mathcal{E}_{i-2}} L_{\mathcal{E}_{i-1}} \mathcal{E}_{i}=: L_{\mathcal{E}_{i-j} \cdots \mathcal{E}_{i-2} \mathcal{E}_{i-1}} \mathcal{E}_{i} .
\end{aligned}
$$

Let $\mathcal{B}=\left(\mathcal{E}_{0}, \ldots, \mathcal{E}_{m}\right)$ be an $m$-block collection of type $\alpha_{0}, \ldots, \alpha_{m}$ of objects of $\mathcal{D}$ which generates $\mathcal{D}$. Two $m$-block collections $\mathcal{H}=\left(\mathcal{H}_{0}, \ldots, \mathcal{H}_{m}\right)$ and $\mathcal{G}=\left(\mathcal{G}_{0}, \ldots, \mathcal{G}_{m}\right)$ of type $\beta_{0}, \ldots, \beta_{m}$ with $\beta_{i}=\alpha_{m-i}$ of objects of $\mathcal{D}$ are called left dual $m$-block collection of $\mathcal{B}$ and right dual $m$-block collection of $\mathcal{B}$ if

$$
\operatorname{Hom}_{\mathcal{D}}^{\bullet}\left(H_{j}^{i}, E_{l}^{k}\right)=\operatorname{Hom}_{\mathcal{D}}^{\bullet}\left(E_{l}^{k}, G_{j}^{i}\right)=0
$$

except for

$$
\operatorname{Ext}_{\mathcal{D}}^{k}\left(H_{i}^{k}, E_{i}^{m-k}\right)=\operatorname{Ext}_{\mathcal{D}}^{m-k}\left(E_{i}^{m-k}, G_{i}^{k}\right)=\mathbb{C}
$$


Remark 3.6. Let $X$ be a smooth projective variety. Given an $m$-block collection $\mathcal{B}=\left(\mathcal{E}_{0}, \ldots, \mathcal{E}_{m}\right)$ of type $\alpha_{0}, \ldots, \alpha_{m}$ of objects of $\mathcal{D}$ which generates $\mathcal{D}$, left dual $m$-block collections and right dual $m$-block collections of $\mathcal{B}$ exist and they are unique up to isomorphism. In fact, by [7]; Proposition 3.9, the $m$-block collection

$$
\mathcal{H}=\left(R^{(0)} \mathcal{E}_{m}, R^{(1)} \mathcal{E}_{m-1}, \ldots, R^{(m)} \mathcal{E}_{0}\right)
$$

where by definition

$$
\begin{aligned}
R^{(i)} \mathcal{E}_{m-i} & =\left(R^{(i)} E_{1}^{m-i}, \ldots, R^{(i)} E_{\alpha_{m-i}}^{m-i}\right) \\
& =\left(R_{\mathcal{E}_{m} \mathcal{E}_{m-1} \cdots \mathcal{E}_{m-i+1}} E_{1}^{m-i}, \ldots, R_{\mathcal{E}_{m} \mathcal{E}_{m-1} \cdots \mathcal{E}_{m-i+1}} E_{\alpha_{m-i}}^{m-i}\right)
\end{aligned}
$$

satisfies the orthogonality conditions (3.5) and (3.6). Therefore, $\mathcal{H}$ is the left dual $m$-block collection of $\mathcal{B}$. By consequent left mutations of the $m$-block collection $\mathcal{B}$ and arguing in the same way we get the right dual $m$-block collection of $\mathcal{B}$.

Let $X$ be an $n$-dimensional smooth projective variety with an $m$-block collection $\mathcal{B}=\left(\mathcal{E}_{0}, \ldots, \mathcal{E}_{m}\right)$ which generates the bounded derived category $\mathcal{D}$. The left dual $m$-block collection of $\mathcal{B}$ will play an important role in our definition of regularity of a coherent sheaf $F$ on $X$ with respect to $\mathcal{B}$ (see Definition 4.5). Therefore, we will now describe explicitly the left dual $m$ block collection of the examples of $m$-block collections given in Example 3.2.

EXAmple 3.7. (1) Let $V$ be a $\mathbb{C}$-vector space of dimension $n+1$ and set $\mathbb{P}^{n}=\mathbb{P}(V)$. We consider the $n$-block collection $\mathcal{B}=\left(\mathcal{O}_{\mathbb{P}^{n}}, \mathcal{O}_{\mathbb{P}^{n}}(1), \ldots\right.$, $\left.\mathcal{O}_{\mathbb{P}^{n}}(n)\right)$ on $\mathbb{P}^{n}$. Using the exterior powers

$$
0 \longrightarrow \bigwedge^{k-1} T_{\mathbb{P}^{n}} \longrightarrow \bigwedge^{k} V \otimes \mathcal{O}_{\mathbb{P}}(k) \longrightarrow \bigwedge^{k} T_{\mathbb{P} n} \longrightarrow 0
$$

of the Euler sequence

$$
0 \longrightarrow \mathcal{O}_{\mathbb{P}^{n}} \longrightarrow V \otimes \mathcal{O}_{\mathbb{P}^{n}}(1) \longrightarrow T_{\mathbb{P}^{n}} \longrightarrow 0
$$

we compute the left dual $n$-block collection of $\mathcal{B}=\left(\mathcal{O}_{\mathbb{P}^{n}}, \mathcal{O}_{\mathbb{P}^{n}}(1), \ldots\right.$, $\left.\mathcal{O}_{\mathbb{P}^{n}}(n)\right)$ and we get

$$
\begin{gathered}
\left(\mathcal{O}_{\mathbb{P}^{n}}(n), R^{(1)} \mathcal{O}_{\mathbb{P}^{n}}(n-1), \ldots, R^{(j)} \mathcal{O}_{\mathbb{P}^{n}}(n-j), \ldots, R^{(n)} \mathcal{O}_{\mathbb{P}^{n}}\right) \\
\quad=\left(\mathcal{O}_{\mathbb{P}^{n}}(n), T_{\mathbb{P}^{n}}(n-1), \ldots, \bigwedge^{j} T_{\mathbb{P}^{n}}(n-j), \ldots, \bigwedge^{n} T_{\mathbb{P}^{n}}\right) .
\end{gathered}
$$


(2) Let $X=\mathbb{P}^{n_{1}} \times \cdots \times \mathbb{P}^{n_{s}}$ be a multiprojective space of dimension $d=n_{1}+\cdots+n_{s}$. For any $0 \leq j \leq d$, denote by $\mathcal{E}_{j}$ the collection of all line bundles on $X$

$$
\mathcal{O}_{X}\left(a_{1}^{j}, a_{2}^{j}, \ldots, a_{s}^{j}\right)
$$

with $-n_{i} \leq a_{i}^{j} \leq 0$ and $\sum_{i=1}^{s} a_{i}^{j}=j-d$. By Example $3.2(4), \mathcal{B}=$ $\left(\mathcal{E}_{0}, \mathcal{E}_{1}, \ldots, \mathcal{E}_{d}\right)$ is a $d$-block collection of line bundles on $X$. By Remark 3.6, the left dual $d$-block collection of $\mathcal{B}$ is given by

$$
\mathcal{H}=\left(R^{(0)} \mathcal{E}_{d}, R^{(1)} \mathcal{E}_{d-1}, \ldots, R^{(d)} \mathcal{E}_{0}\right)
$$

where by definition

$$
\begin{aligned}
R^{(k)} \mathcal{E}_{d-k} & =\left(\ldots, R^{(k)} \mathcal{O}_{X}\left(t_{1}, \ldots, t_{s}\right), \ldots\right) \\
& =\left(\ldots, R_{\mathcal{E}_{d} \cdots \mathcal{E}_{d-k+1}} \mathcal{O}_{X}\left(t_{1}, \ldots, t_{s}\right), \ldots\right)
\end{aligned}
$$

A straightforward computation shows that for any $\mathcal{O}_{X}\left(t_{1}, \ldots, t_{s}\right) \in \mathcal{E}_{d-k}$ and any $0 \leq k \leq d$,

$$
\begin{aligned}
R^{(k)} \mathcal{O}_{X}\left(t_{1}, \ldots, t_{s}\right)= & R_{\mathcal{E}_{d} \cdots \mathcal{E}_{d-k+1}} \mathcal{O}_{X}\left(t_{1}, \ldots, t_{s}\right) \\
& =\bigwedge^{-t_{1}} T_{\mathbb{P}^{n_{1}}}\left(t_{1}\right) \otimes \cdots \otimes \bigwedge^{-t_{s}} T_{\mathbb{P}^{n_{s}}}\left(t_{s}\right)
\end{aligned}
$$

(3) Let $Q_{n} \subset \mathbb{P}^{n+1}, n \geq 2$, be a hyperquadric variety and let $\mathcal{B}=$ $\left(\mathcal{E}_{0}, \mathcal{E}_{1}, \ldots, \mathcal{E}_{n}\right)$ where

$$
\mathcal{E}_{j}=\mathcal{O}_{Q_{n}}(-n+j) \quad \text { for } 1 \leq j \leq n, \quad \mathcal{E}_{0}= \begin{cases}\left(\Sigma_{1}(-n), \Sigma_{2}(-n)\right) & \text { if } n \text { even } \\ (\Sigma(-n)) & \text { if } n \text { odd }\end{cases}
$$

be the $n$-block collection of locally free sheaves on $Q_{n}$ described in Example $3.2(3)$. To define the left dual $n$-block collection of $\mathcal{B}$ we need to fix some notation. We set $\Omega^{j}:=\Omega_{\mathbb{P}^{n+1}}^{j}$ and we define inductively $\psi_{j}$ :

$$
\psi_{0}:=\mathcal{O}_{Q_{n}}, \quad \psi_{1}:=\Omega^{1}(1)_{\mid Q_{n}}
$$

and, for all $j \geq 2$, we define the locally free sheaf $\psi_{j}$ as the unique nonsplitting extension (note that $\operatorname{Ext}^{1}\left(\psi_{j-2}, \Omega^{j}(j)_{\mid Q_{n}}\right)=\mathbb{C}$ ):

$$
0 \longrightarrow \Omega^{j}(j)_{\mid Q_{n}} \longrightarrow \psi_{j} \longrightarrow \psi_{j-2} \longrightarrow 0
$$

By [16]; Proposition 4.11 and using the fact that the left dual $n$-block collection of a given $n$-block collection is uniquely determined up to unique 
isomorphism by the orthogonality conditions described in Remark 3.6, we get that the left dual $n$-block collection of the $n$-block collection $\mathcal{B}$ is

$$
\mathcal{H}=\left(R^{(0)} \mathcal{E}_{n}, R^{(1)} \mathcal{E}_{n-1}, \ldots, R^{(n)} \mathcal{E}_{0}\right)
$$

where

$$
R^{(j)} \mathcal{E}_{n-j}=\psi_{j}^{*} \quad \text { for } 1 \leq j \leq n, \quad R^{(n)} \mathcal{E}_{0}= \begin{cases}\left(\Sigma_{1}^{*}(1), \Sigma_{2}^{*}(1)\right) & \text { if } n \text { even } \\ \left(\Sigma^{*}(1)\right) & \text { if } n \text { odd } .\end{cases}
$$

(4) Let $X=G r(k, n)$ be the Grassmannian of $k$-dimensional subspaces of the $n$-dimensional vector space and let $\mathcal{B}=\left(\mathcal{E}_{0}, \mathcal{E}_{1}, \ldots, \mathcal{E}_{k(n-k)}\right)$ with $\mathcal{E}_{r}=\left\{\Sigma^{\alpha} \mathcal{S}|| \alpha \mid=k(n-k)-r\right\}$ be the $k(n-k)$-block collection of locally free sheaves on $X$ described in Example 3.2 (2). The sequence $\alpha$ defines a Young diagram and we denote by $\tilde{\alpha}$ the sequence corresponding to the conjugate diagram. By [16] Lemma 3.2, for any two indices: $\alpha: n-k \geq$ $\alpha_{1} \geq \cdots \geq \alpha_{k} \geq 0$ and $\beta: k \geq \beta_{1} \geq \cdots \geq \beta_{n-k} \geq 0$ we have

$$
H^{i}\left(X, \Sigma^{\alpha} \mathcal{S} \otimes \Sigma^{\beta} \mathcal{Q}^{*}\right)= \begin{cases}\mathbb{C} & \text { if } \alpha=\tilde{\beta} \text { and } i=|\alpha| \\ 0 & \text { otherwise. }\end{cases}
$$

Therefore, the left dual $k(n-k)$-block collection of $\mathcal{B}=\left(\mathcal{E}_{0}, \mathcal{E}_{1}, \ldots\right.$, $\left.\mathcal{E}_{k(n-k)}\right)$ with $\mathcal{E}_{r}=\left\{\Sigma^{\alpha} \mathcal{S}|| \alpha \mid=k(n-k)-r\right\}$ is

$$
\mathcal{H}=\left(R^{(0)} \mathcal{E}_{k(n-k)}, R^{(1)} \mathcal{E}_{k(n-k)-1}, \ldots, R^{(j)} \mathcal{E}_{k(n-k)-j}, \ldots, R^{(k(n-k))} \mathcal{E}_{0}\right)
$$

where $R^{(r)} \mathcal{E}_{k(n-k)-r}=\left\{\Sigma^{\tilde{\alpha}} \mathcal{Q}|| \alpha \mid=r\right\}$.

We want to point out that the notion of $m$-block collection is the convenient generalization of the notion of geometric collection we were looking for. Indeed, we will see that the behavior of $n$-block collections, $n=\operatorname{dim}(X)$, is really good in the sense that they are automatically strongly exceptional collections and that their structure is preserved under mutations through blocks. More precisely we have:

Proposition 3.8. Let $X$ be a smooth projective variety of dimension $n$ and let $\mathcal{B}=\left(\mathcal{E}_{0}, \ldots, \mathcal{E}_{n}\right)$ be an $n$-block collection of coherent sheaves on $X$ and assume that $\mathcal{B}$ generates the category $\mathcal{D}$. Then, we have:

(1) The sequence $\mathcal{B}$ is a full strongly exceptional collection of coherent sheaves on $X$. 
(2) All mutations through the blocks $\mathcal{E}_{j}$ can be computed using short exact sequences of coherent sheaves.

(3) Any mutation of $\mathcal{B}$ through any block $\mathcal{E}_{j}$ is a full strongly exceptional collection of pure sheaves, i.e. complexes concentrated in the zero component of the grading.

(4) Any mutation of $\mathcal{B}$ through any block $\mathcal{E}_{j}$ is an $n$-block collection.

Proof. See [2]; Theorem 9.5 and Remark b) below and [13]; Theorem 1.

Remark 3.9. By Remark 2.7 (2), a mutation of a strongly exceptional collection is not, in general, a strongly exceptional collection. In fact, $\sigma=\left(\mathcal{O}_{X}, \mathcal{O}_{X}(1,0), \mathcal{O}_{X}(0,1), \mathcal{O}_{X}(1,1)\right)$ is a strongly exceptional collection on $X=\mathbb{P}^{1} \times \mathbb{P}^{1}$ and the mutated collection

$$
\begin{gathered}
\left(\mathcal{O}_{X}, \mathcal{O}_{X}(1,0), L_{\mathcal{O}_{X}(0,1)} \mathcal{O}_{X}(1,1), \mathcal{O}_{X}(0,1)\right) \\
=\left(\mathcal{O}_{X}, \mathcal{O}_{X}(1,0), \mathcal{O}_{X}(-1,1), \mathcal{O}_{X}(0,1)\right)
\end{gathered}
$$

is no more a strongly exceptional collection on $X$. However, we can pack the objects of $\sigma$ in a suitable subcollections of blocks $\mathcal{B}=\left(\mathcal{E}_{0}, \mathcal{E}_{1}, \mathcal{E}_{2}\right)=$ $\left(\mathcal{O}_{X},\left(\mathcal{O}_{X}(1,0), \mathcal{O}_{X}(0,1)\right), \mathcal{O}_{X}(1,1)\right)$ and according to Proposition 3.8 any mutation of $\mathcal{B}$ through any block $\mathcal{E}_{j}$ is a full strongly exceptional collection. So, for instance, the mutation of $\mathcal{B}$ through the block $\mathcal{E}_{1}$ is the full strongly exceptional collection

$$
\begin{aligned}
\left(\mathcal{E}_{0}, L_{\mathcal{E}_{1}} \mathcal{E}_{2}, \mathcal{E}_{1}\right) & =\left(\mathcal{O}_{X}, L_{\mathcal{O}_{X}(1,0) \mathcal{O}_{X}(0,1)} \mathcal{O}_{X}(1,1),\left(\mathcal{O}_{X}(1,0), \mathcal{O}_{X}(0,1)\right)\right. \\
& =\left(\mathcal{O}_{X}, T_{X}(-1,-1),\left(\mathcal{O}_{X}(1,0), \mathcal{O}_{X}(0,1)\right)\right)
\end{aligned}
$$

To compute $L_{\mathcal{E}_{1}} \mathcal{E}_{2}=L_{\mathcal{O}_{X}(1,0) \mathcal{O}_{X}(0,1)} \mathcal{O}_{X}(1,1)=T_{X}(-1,-1)$ we have used the exact sequences

$$
\begin{aligned}
& 0 \longrightarrow \mathcal{O}_{X}(-1,1) \longrightarrow V^{*} \otimes \mathcal{O}_{X}(0,1) \longrightarrow \mathcal{O}_{X}(1,1) \longrightarrow 0, \quad \text { and } \\
& 0 \longrightarrow \mathcal{O}_{X}(-1,1) \longrightarrow T_{X}(-1,-1) \longrightarrow \wedge^{2} V^{*} \otimes \mathcal{O}_{X}(1,0) \longrightarrow 0
\end{aligned}
$$

being $X=\mathbb{P}^{1} \times \mathbb{P}^{1}$ and $\mathbb{P}^{1}=\mathbb{P}(V)$.

Beilinson Theorem was stated in 1978 [1] and since then it has became a major tool in classifying vector bundles over projective spaces. Beilinson spectral sequence was generalized by Kapranov to hyperquadrics and 
Grassmannians ([15] and [16]) and by the authors to any smooth projective variety with a geometric collection [6]. We are now ready to generalize Beilinson Theorem to any smooth projective variety $X$ of dimension $n$ with an $n$-block collection $\mathcal{B}=\left(\mathcal{E}_{0}, \mathcal{E}_{1}, \ldots, \mathcal{E}_{n}\right), \mathcal{E}_{j}=\left(E_{1}^{j}, \ldots, E_{\alpha_{j}}^{j}\right)$ of coherent sheaves on $X$ which generates $\mathcal{D}$.

Theorem 3.10. (Beilinson type spectral sequence) Let $X$ be a smooth projective variety of dimension $n$ with an n-block collection $\mathcal{B}=\left(\mathcal{E}_{0}, \mathcal{E}_{1}, \ldots\right.$, $\left.\mathcal{E}_{n}\right), \mathcal{E}_{i}=\left(E_{1}^{i}, \ldots, E_{\alpha_{i}}^{i}\right)$ of coherent sheaves on $X$ which generates $\mathcal{D}$. Then for any coherent sheaf $F$ on $X$ there are two spectral sequences situated in the square $-n \leq p \leq 0,0 \leq q \leq n$, with $E_{1}$-term

$$
\begin{aligned}
{ }_{I} E_{1}^{p q} & = \begin{cases}\bigoplus_{i=1}^{\alpha_{p+n}} \operatorname{Ext}^{q}\left(R_{\mathcal{E}_{n} \cdots \mathcal{E}_{p+n+1}} E_{i}^{p+n}, F\right) \otimes E_{i}^{p+n} \\
\bigoplus_{i=1}^{\alpha_{n}} \operatorname{Ext}^{q}\left(E_{i}^{n}, F\right) \otimes E_{i}^{n} & \text { if }-n \leq p \leq-1\end{cases} \\
{ }_{I} E_{1}^{p q} & = \begin{cases}\bigoplus_{i=1}^{\alpha_{p+n}} \operatorname{Ext}^{q}\left(\left(E_{i}^{p+n}\right)^{*}, F\right) \otimes\left(R_{\mathcal{E}_{n} \cdots \mathcal{E}_{p+n+1}} E_{i}^{p+n}\right)^{*} \\
\bigoplus_{i=1}^{\alpha_{n}} \operatorname{Ext}^{q}\left(E_{i}^{n *}, F\right) \otimes E_{i}^{n *} & \text { if }-n \leq p \leq-1\end{cases}
\end{aligned}
$$

and differentials $d_{r}^{p q}: E_{r}^{p, q} \rightarrow E_{r}^{p+r, q-r+1}$ which converge to

$$
{ }_{I} E_{\infty}^{i}={ }_{I I} E_{\infty}^{i}= \begin{cases}F & \text { for } i=0 \\ 0 & \text { for } i \neq 0 .\end{cases}
$$

Proof. We will only prove the existence of the first spectral sequence. The other can be done similarly. For any $\gamma, 0 \leq \gamma \leq n$, we write ${ }^{i} V_{\gamma}^{\bullet}$ for the graded vector spaces

$$
{ }^{i} V_{\gamma}^{\bullet}=\operatorname{Hom}_{\mathcal{D}}^{\bullet}\left(R_{\mathcal{E}_{n} \cdots \mathcal{E}_{\gamma+1}} E_{i}^{\gamma}, F\right)=\operatorname{Hom}_{\mathcal{D}}^{\bullet}\left(E_{i}^{\gamma}, L_{\mathcal{E}_{\gamma+1} \cdots \mathcal{E}_{n}} F\right)
$$

where the second equality follows from standard properties of mutations ([11]; Pag. 12-14).

By Remark 3.4, the triangles defining the consequent right mutations of $F$ and the consequent left mutations of $F[n]$ through $\left(\mathcal{E}_{0}, \ldots, \mathcal{E}_{n}\right)$ can be written as

$$
\begin{gathered}
\left(\bigoplus_{i=1}^{\alpha_{\gamma}} i V_{\gamma}^{\bullet} \otimes E_{i}^{\gamma}\right)[-1] \stackrel{k_{\gamma}}{\longrightarrow} R_{\mathcal{E}_{\gamma} \cdots \mathcal{E}_{0}} F[-1] \stackrel{i_{\gamma}}{\longrightarrow} R_{\mathcal{E}_{\gamma-1} \cdots \mathcal{E}_{0}} F \stackrel{j_{\gamma}}{\longrightarrow} \bigoplus_{i=1}^{\alpha_{\gamma}} i V_{\gamma}^{\bullet} \otimes E_{i}^{\gamma} \\
\bigoplus_{i=1}^{\alpha_{\gamma}} V_{\gamma}^{\bullet} \otimes E_{i}^{\gamma} \stackrel{j^{\gamma+1}}{\longrightarrow} L_{\mathcal{E}_{\gamma+1} \cdots \mathcal{E}_{n}} F[n] \stackrel{i^{\gamma+1}}{\longrightarrow} L_{\mathcal{E}_{\gamma} \cdots \mathcal{E}_{n}} F[n+1] \stackrel{k^{\gamma+1}}{\longrightarrow}\left(\bigoplus_{i=1}^{\alpha_{\gamma}} V_{\gamma}^{\bullet} \otimes E_{i}^{\gamma}\right)[1] .
\end{gathered}
$$


We arrange them into the following big diagram:

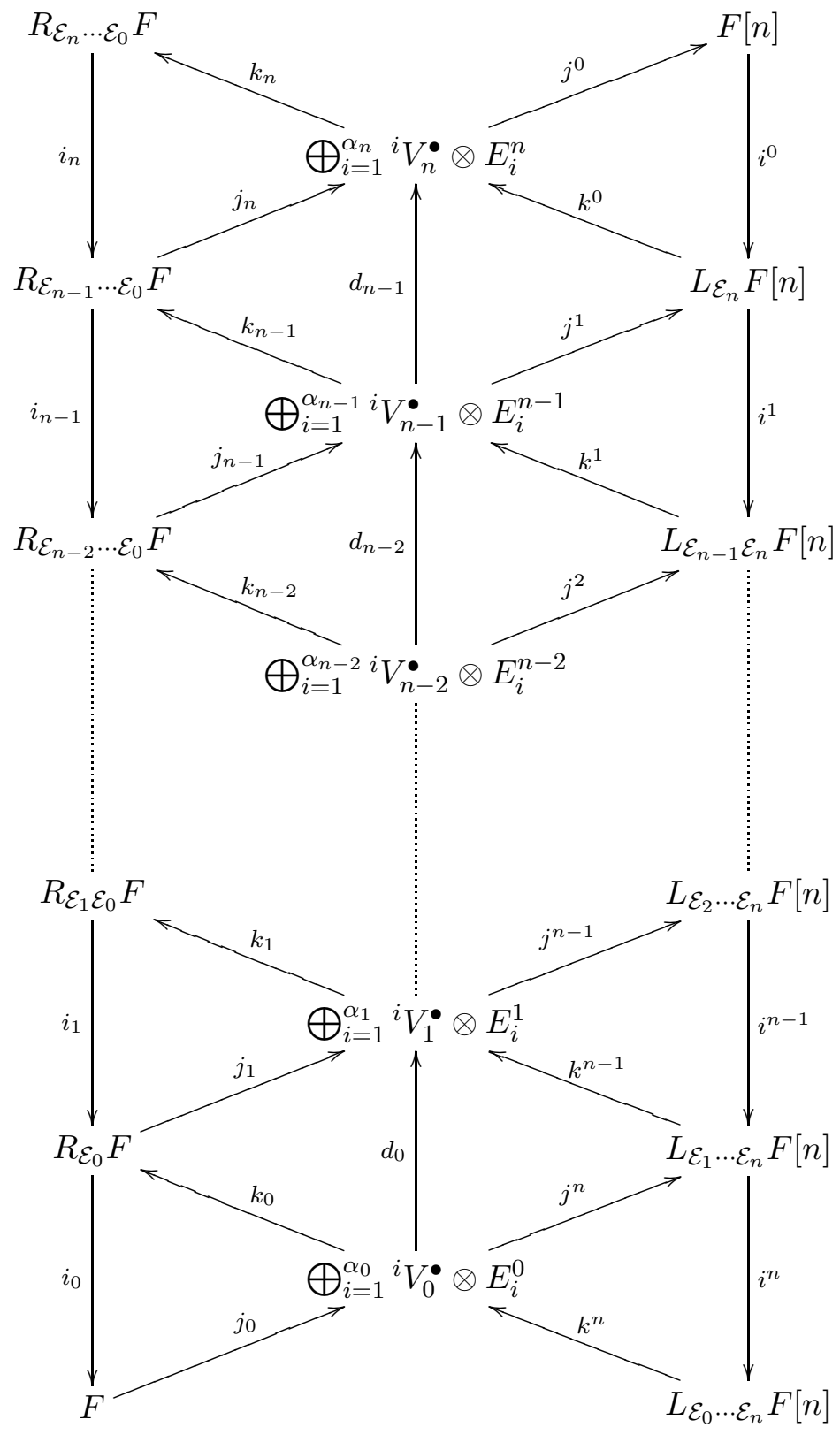

At this diagram, all oriented triangles along left and right vertical borders are distinguished, the morphisms $i \bullet$ and $i^{\bullet}$ have degree one, and all 
triangles and rhombuses in the central column are commutative. So, there is the following complex, functorial on $F$,

$$
\begin{aligned}
L^{\bullet}: 0 \longrightarrow \bigoplus_{i=1}^{\alpha_{0}}{ }^{i} V_{0}^{\bullet} \otimes E_{i}^{0} \longrightarrow \bigoplus_{i=1}^{\alpha_{1}}{ }^{i} V_{1}^{\bullet} \otimes E_{i}^{1} \longrightarrow \cdots \\
\cdots \longrightarrow \bigoplus_{i=1}^{\alpha_{n-1} i} V_{n-1}^{\bullet} \otimes E_{i}^{n-1} \longrightarrow \bigoplus_{i=1}^{\alpha_{n}}{ }^{i} V_{n}^{\bullet} \otimes E_{i}^{n} \longrightarrow 0
\end{aligned}
$$

and by the above Postnikov-system we have that $F$ is a right convolution of this complex. Then, for an arbitrary linear covariant cohomological functor $\Phi^{\bullet}$, there exists an spectral sequence with $E_{1}$-term

$$
{ }_{I} E_{1}^{p q}=\Phi^{q}\left(L^{p}\right)
$$

situated in the square $0 \leq p, q \leq n$ and converging to $\Phi^{p+q}(F)$ (see [16]; 1.5). Since $\Phi^{\bullet}$ is a linear functor, we have

$$
\begin{aligned}
\Phi^{q}\left(L^{p}\right) & =\bigoplus_{i=1}^{\alpha_{p}} \Phi^{q}\left({ }^{i} V_{p}^{\bullet} \otimes E_{i}^{p}\right)=\bigoplus_{i=1}^{\alpha_{p}} \bigoplus_{l}{ }^{i} V_{p}^{l} \otimes \Phi^{q-l}\left(E_{i}^{p}\right) \\
& =\bigoplus_{i=1}^{\alpha_{p}} \bigoplus_{\alpha+\beta=q}{ }^{i} V_{p}^{\alpha} \otimes \Phi^{\beta}\left(E_{i}^{p}\right)
\end{aligned}
$$

In particular, if we consider the covariant linear cohomology functor which takes a complex to its cohomology sheaf and acts identically on pure sheaves, i.e.

$$
\Phi^{\beta}(F)= \begin{cases}F & \text { for } \beta=0 \\ 0 & \text { for } \beta \neq 0\end{cases}
$$

on any pure sheaf $F$, in the square $0 \leq p, q \leq n$, we get

$$
{ }_{I} E_{1}^{p q}=\bigoplus_{i=1}^{\alpha_{p}}{ }^{i} V_{p}^{q} \otimes E_{i}^{p}=\bigoplus_{i=1}^{\alpha_{p}} \operatorname{Ext}^{q}\left(R_{\mathcal{E}_{n} \cdots \mathcal{E}_{p+1}} E_{i}^{p}, F\right) \otimes E_{i}^{p}
$$

which converges to

$$
{ }_{I} E_{\infty}^{i}= \begin{cases}F & \text { for } i=0 \\ 0 & \text { for } i \neq 0 .\end{cases}
$$

Finally, if we call $p^{\prime}=p-n$, we get the spectral sequence

$$
{ }_{I} E_{1}^{p^{\prime} q}=\bigoplus_{i=1}^{\alpha_{p^{\prime}+n}} \operatorname{Ext}^{q}\left(R_{\mathcal{E}_{n} \cdots \mathcal{E}_{p^{\prime}+n+1}} E_{i}^{p^{\prime}+n}, F\right) \otimes E_{i}^{p^{\prime}+n}
$$


situated in the square $-n \leq p^{\prime} \leq 0,0 \leq q \leq n$ which converges to

$$
{ }_{I} E_{\infty}^{i}= \begin{cases}F & \text { for } i=0 \\ 0 & \text { for } i \neq 0 .\end{cases}
$$

Remark 3.11. We want to point out that in Theorem 3.10 the number of blocks is one greater than the dimension of $X$ but a priori there is no restriction on the length $\alpha_{j}$ of each block $\mathcal{E}_{j}=\left(E_{1}^{j}, \ldots, E_{\alpha_{j}}^{j}\right)$.

Arguing as in [6]; Lemma 2.23 but using the distinguished triangles (3.1) and (3.2) instead of exact sequences, we can prove the following technical Lemma that will be used in next sections.

LEMMA 3.12. Let $X$ be a smooth projective variety of dimension $n$ and let $\mathcal{B}=\left(\mathcal{E}_{0}, \ldots, \mathcal{E}_{n}\right)$ be an $n$-block collection of coherent sheaves on $X$. For any $i<j$ and any locally free sheaf $F$ on $X$, it holds:

(a) $\left(L_{\mathcal{E}_{i}} E_{k}^{j}\right)^{*}=R_{\mathcal{E}_{i}^{*}} E_{k}^{j *}$ for any $E_{k}^{j} \in \mathcal{E}_{j}$;

(b) $\left(R_{\mathcal{E}_{j}} E_{k}^{i}\right)^{*}=L_{\mathcal{E}_{j}^{*}} E_{k}^{i *}$ for any $E_{k}^{i} \in \mathcal{E}_{i}$;

(c) $\left(R_{\mathcal{E}_{j}} E_{k}^{i}\right) \otimes F \cong R_{\mathcal{E}_{j} \otimes F}\left(E_{k}^{i} \otimes F\right)$ for any $E_{k}^{i} \in \mathcal{E}_{i}$ and $\left(L_{\mathcal{E}_{i}} E_{k}^{j}\right) \otimes F \cong$ $L_{\mathcal{E}_{i} \otimes F}\left(E_{k}^{j} \otimes F\right)$ for any $E_{k}^{j} \in \mathcal{E}_{j}$.

\section{§4. Regularity with respect to $n$-blocks collections}

The goal of this section is to extend the notion of Castelnuovo-Mumford regularity for coherent sheaves on a projective space to coherent sheaves on an $n$-dimensional smooth projective variety with an $n$-block collection of coherent sheaves on $X$ which generates $\mathcal{D}$. We establish for coherent sheaves on $\mathbb{P}^{n}$ the agreement of the new definition of regularity with the old one and we prove that many formal properties of Castelnuovo-Mumford regularity continue to hold in our more general setup.

To extend the notion of Castelnuovo-Mumford regularity, we will first introduce the notion of helix of blocks associated to an $m$-block collection of objects of $\mathcal{D}$ as a natural generalization of the notion of helix associated to an exceptional collection of objects of $\mathcal{D}$ introduced by J. M. Drezet and J. Le Potier in [8] and L. Gorodentsev and A. N. Rudakov in [12] (see also $[13])$.

Definition 4.1. Let $X$ be a smooth projective variety and let $\mathcal{B}=$ $\left(\mathcal{E}_{0}, \mathcal{E}_{1}, \ldots, \mathcal{E}_{m}\right), \mathcal{E}_{j}=\left(E_{1}^{j}, \ldots, E_{\alpha_{j}}^{j}\right)$ be an $m$-block collection of objects of 
$\mathcal{D}$. We extend, in both directions, the collection $\mathcal{B}$ to an infinite sequence of blocks defining by induction:

$$
\mathcal{E}_{i+m}:=R^{(m)} \mathcal{E}_{i-1} \quad \text { and } \quad \mathcal{E}_{-i}=L^{(m)} \mathcal{E}_{m-i+1} \quad i>0 .
$$

The collection $\mathcal{H}_{\mathcal{B}}=\left\{\mathcal{E}_{i}\right\}_{i \in \mathbb{Z}}$ is called the helix of blocks associated to $\mathcal{B}$. Each helix $\mathcal{H}_{\mathcal{B}}=\left\{\mathcal{E}_{i}\right\}_{i \in \mathbb{Z}}$ is uniquely recovered from any collection of $(m+1)$ of its consequent blocks $\mathcal{B}_{i}=\left(\mathcal{E}_{i}, \mathcal{E}_{i+1}, \ldots, \mathcal{E}_{i+m}\right)$.

Remark 4.2. Let $X$ be a smooth projective variety, let $\mathcal{B}=\left(\mathcal{E}_{0}, \mathcal{E}_{1}, \ldots\right.$, $\left.\mathcal{E}_{m}\right), \mathcal{E}_{j}=\left(E_{1}^{j}, \ldots, E_{\alpha_{j}}^{j}\right)$, be an $m$-block collection and let $\mathcal{H}_{\mathcal{B}}=\left\{\mathcal{E}_{i}\right\}_{i \in \mathbb{Z}}$ be the helix of blocks associated to $\mathcal{B}$. If we consider $\mathcal{H}_{\mathcal{B}}$ just as a collection of objects of $\mathcal{D}$ (we forget the blocks) then it turns out to be the helix associated to the exceptional collection

$$
\sigma=\left(\mathcal{E}_{0}, \mathcal{E}_{1}, \ldots, \mathcal{E}_{m}\right)=\left(E_{1}^{0}, \ldots, E_{\alpha_{0}}^{0}, E_{1}^{1}, \ldots, E_{\alpha_{1}}^{1}, \ldots, E_{1}^{m}, \ldots, E_{\alpha_{m}}^{m}\right) .
$$

Definition 4.3. Let $X$ be a smooth projective variety of dimension $n$ with canonical bundle $K_{X}$. A sequence $\left\{\mathcal{E}_{i}\right\}_{i \in \mathbb{Z}}$ of blocks of objects of $\mathcal{D}$ will be called a helix of blocks of period $(m+1)$ if for any $i \in \mathbb{Z}$,

$$
\mathcal{E}_{i}=\mathcal{E}_{i+m+1} \otimes K_{X}[n-m]
$$

where $\mathcal{E}_{j} \otimes K_{X}[n-m]$ denotes the block $\left(E_{1}^{j} \otimes K_{X}[n-m], E_{2}^{j} \otimes K_{X}[n-\right.$ $\left.m], \ldots, E_{\alpha_{j}}^{j} \otimes K_{X}[n-m]\right)$ and the number in square brackets denotes the multiplicity of the shift of an object to the left viewed as a graded complex in $\mathcal{D}$.

It follows from Remark 4.2 and [2]; Theorem 4.1 that if $X$ is a smooth projective variety of dimension $n$ and $\mathcal{B}=\left(\mathcal{E}_{0}, \mathcal{E}_{1}, \ldots, \mathcal{E}_{m}\right), \mathcal{E}_{j}=\left(E_{1}^{j}, \ldots\right.$, $\left.E_{\alpha_{j}}^{j}\right)$, is an $m$-block collection of objects of $\mathcal{D}$, then the helix of blocks $\mathcal{H}_{\mathcal{B}}=\left\{\mathcal{E}_{i}\right\}_{i \in \mathbb{Z}}$ associated to $\mathcal{B}$ is an helix of blocks of period $m+1$, i.e., for any $i \in \mathbb{Z}, \mathcal{E}_{i}=\mathcal{E}_{i+m+1} \otimes K_{X}[n-m]$.

As an immediate consequence of Proposition 3.8, we have:

Corollary 4.4. Let $X$ be a smooth projective variety of dimension n, let $\mathcal{B}=\left(\mathcal{E}_{0}, \ldots, \mathcal{E}_{n}\right), \mathcal{E}_{j}=\left(E_{1}^{j}, \ldots, E_{\alpha_{j}}^{j}\right)$ be an $n$-block collection of coherent sheaves on $X$ which generates $\mathcal{D}$ and denote by $\mathcal{H}_{\mathcal{B}}=\left\{\mathcal{E}_{i}\right\}_{i \in \mathbb{Z}}$ the helix of blocks associated to $\mathcal{B}$. Then, $\mathcal{H}_{\mathcal{B}}$ is an helix of period $n+1$ and any $n$-block collection $\mathcal{B}_{i}=\left(\mathcal{E}_{i}, \mathcal{E}_{i+1}, \ldots, \mathcal{E}_{i+n}\right)$ of $n+1$ subsequent blocks is an $n$-block collection of coherent sheaves on $X$ which generates $\mathcal{D}$. 
Let $X$ be a smooth projective variety of dimension $n$ and let $\mathcal{B}=$ $\left(\mathcal{E}_{0}, \mathcal{E}_{1}, \ldots, \mathcal{E}_{n}\right), \mathcal{E}_{j}=\left(E_{1}^{j}, \ldots, E_{\alpha_{j}}^{j}\right)$ be an $n$-block collection of coherent sheaves on $X$ which generates $\mathcal{D}$. Associated to $\mathcal{B}$ we have a helix of blocks $\mathcal{H}_{\mathcal{B}}=\left\{\mathcal{E}_{i}\right\}_{i \in \mathbb{Z}}$; and for any $n$-block collection $\mathcal{B}_{i}=\left(\mathcal{E}_{i}, \mathcal{E}_{i+1}, \ldots, \mathcal{E}_{i+n}\right)$ of $n+1$ subsequent blocks and any coherent $\mathcal{O}_{X}$-module $F$ we have a spectral sequence (see Theorem 3.10)

$$
{ }_{I}^{i} E_{1}^{p q}= \begin{cases}\bigoplus_{s=1}^{\alpha_{p+n+i}} \operatorname{Ext}^{q}\left(R_{\mathcal{E}_{i+n} \cdots \mathcal{E}_{i+p+n+1}} E_{s}^{i+p+n}, F\right) \otimes E_{s}^{i+p+n} \\ \bigoplus_{s=1}^{\alpha_{i+n}} \operatorname{Ext}^{q}\left(E_{s}^{i+n}, F\right) \otimes E_{s}^{i+n} & \text { if }-n \leq p \leq-1\end{cases}
$$

situated in the square $0 \leq q \leq n,-n \leq p \leq 0$ which converges to

$$
E_{\infty}^{r}= \begin{cases}F & \text { for } r=0 \\ 0 & \text { for } r \neq 0\end{cases}
$$

DEFINITION 4.5. Let $X$ be a smooth projective variety of dimension $n$ with an $n$-block collection $\mathcal{B}=\left(\mathcal{E}_{0}, \mathcal{E}_{1}, \ldots, \mathcal{E}_{n}\right), \mathcal{E}_{i}=\left(E_{1}^{i}, \ldots, E_{\alpha_{i}}^{i}\right)$ of coherent sheaves on $X$ which generates $\mathcal{D}$, let $\mathcal{H}_{\mathcal{B}}=\left\{\mathcal{E}_{i}\right\}_{i \in \mathbb{Z}}$ be the helix of blocks associated to $\mathcal{B}$ and let $F$ be a coherent $\mathcal{O}_{X}$-module. We say that $F$ is $m$-regular with respect to $\mathcal{B}$ if for $q>0$ we have

$$
\begin{cases}\bigoplus_{s=1}^{\alpha_{-m+p}} \operatorname{Ext}^{q}\left(R_{\left.\mathcal{E}_{-m} \cdots \mathcal{E}_{-m+p+1} E_{s}^{-m+p}, F\right)=0}\right. & \text { for }-n \leq p \leq-1 \\ \bigoplus_{s=1}^{\alpha_{-m}} \operatorname{Ext}^{q}\left(E_{s}^{-m}, F\right)=0 & \text { for } p=0 .\end{cases}
$$

So, $F$ is $m$-regular with respect to $\mathcal{B}$ if ${ }^{-n-m} E_{1}^{p q}=0$ for $q>0$ in (4.1). In particular, if $F$ is $m$-regular with respect to $\mathcal{B}$ the spectral sequence ${ }^{-n-m} E_{1}^{p q}$ collapses at $E_{2}$ and we get the following exact sequence:

$$
0 \longrightarrow \mathcal{L}_{-n} \longrightarrow \cdots \longrightarrow \mathcal{L}_{-1} \longrightarrow \mathcal{L}_{0} \longrightarrow F \longrightarrow 0
$$

where

$$
\mathcal{L}_{p}= \begin{cases}\bigoplus_{s=1}^{\alpha_{-m+p}} H^{0}\left(X,\left(R_{\mathcal{E}_{-m} \cdots \mathcal{E}_{-m+p+1}} E_{s}^{-m+p}\right)^{*} \otimes F\right) \otimes E_{s}^{-m+p} \\ \bigoplus_{s=1}^{\alpha_{-m}} H^{0}\left(\left(E_{s}^{-m}\right)^{*} \otimes F\right) \otimes E_{s}^{-m} & \text { if }-n \leq p \leq-1\end{cases}
$$

Definition 4.6. Let $X$ be a smooth projective variety of dimension $n$ with an $n$-block collection $\mathcal{B}=\left(\mathcal{E}_{0}, \mathcal{E}_{1}, \ldots, \mathcal{E}_{n}\right), \mathcal{E}_{j}=\left(E_{1}^{j}, \ldots, E_{\alpha_{j}}^{j}\right)$ of 
coherent sheaves on $X$ which generates $\mathcal{D}$ and let $F$ be a coherent $\mathcal{O}_{X^{-}}$ module. We define the regularity of $F$ with respect to $\mathcal{B}$ (or $\mathcal{B}$-regularity of $F), \operatorname{Reg}_{\mathcal{B}}(F)$, as the least integer $m$ such that $F$ is $m$-regular with respect to $\mathcal{B}$ in the sense of Definition 4.5. We set $\operatorname{Reg}_{\mathcal{B}}(F)=-\infty$ if there is no such integer.

Remark 4.7. Let $X$ be a smooth projective variety of dimension $n$. Since a geometric collection $\sigma=\left(E_{0}, E_{1}, \ldots, E_{n}\right)$ is an $n$-block collection of type $(1, \ldots, 1)$, Definitions 4.5 and 4.6 extend the definition of regularity with respect to a geometric collection introduced by the authors in [6].

EXAMPLE 4.8. We consider the $n$-block collection $\mathcal{B}=\left(\mathcal{O}_{\mathbb{P}^{n}}, \mathcal{O}_{\mathbb{P}^{n}}(1)\right.$, $\left.\ldots, \mathcal{O}_{\mathbb{P} n}(n)\right)$ on $\mathbb{P}^{n}$ and the associated helix $\mathcal{H}_{\mathcal{B}}=\left\{\mathcal{O}_{\mathbb{P}^{n}}(i)\right\}_{i \in \mathbb{Z}}$. According to Example 3.7 (1), the left dual $n$-block collection of an $n$-block collection $\mathcal{B}_{i}=\left(\mathcal{O}_{\mathbb{P}^{n}}(i), \mathcal{O}_{\mathbb{P}^{n}}(i+1), \ldots, \mathcal{O}_{\mathbb{P}^{n}}(i+n)\right)$ of $n+1$ subsequent blocks of $\mathcal{H}_{\mathcal{B}}$ is

$$
\begin{gathered}
\left(\mathcal{O}_{\mathbb{P}^{n}}(i+n), R^{(1)} \mathcal{O}_{\mathbb{P}^{n}}(i+n-1), \ldots, R^{(j)} \mathcal{O}_{\mathbb{P}^{n}}(i+n-j), \ldots, R^{(n)} \mathcal{O}_{\mathbb{P}^{n}}(i)\right) \\
\quad=\left(\mathcal{O}_{\mathbb{P}^{n}}(i+n), T_{\mathbb{P}^{n}}(i+n-1), \ldots, \bigwedge^{j} T_{\mathbb{P}^{n}}(i+n-j), \ldots, \bigwedge^{n} T_{\mathbb{P}^{n}}(i)\right) .
\end{gathered}
$$

Therefore, for any coherent sheaf $F$ on $\mathbb{P}^{n}$ our definition reduces to say: $F$ is $m$-regular with respect to $\mathcal{B}$ if $\operatorname{Ext}^{q}\left(\bigwedge^{-p} T(-m+p), F\right)=H^{q}\left(\mathbb{P}^{n}, \Omega^{-p}(m-\right.$ $p) \otimes F)=0$ for all $q>0$ and all $p,-n \leq p \leq 0$.

We will now compute the regularity with respect to $\mathcal{B}=\left(\mathcal{E}_{0}, \mathcal{E}_{1}, \ldots, \mathcal{E}_{n}\right)$ of any coherent sheaf $E_{t}^{i} \in \mathcal{E}_{i}$.

Proposition 4.9. Let $X$ be a smooth projective variety of dimension $n$ with an $n$-block collection $\mathcal{B}=\left(\mathcal{E}_{0}, \mathcal{E}_{1}, \ldots, \mathcal{E}_{n}\right), \mathcal{E}_{j}=\left(E_{1}^{j}, \ldots, E_{\alpha_{j}}^{j}\right)$, which generates $\mathcal{D}$ and let $\mathcal{H}_{\mathcal{B}}=\left\{\mathcal{E}_{i}\right\}_{i \in \mathbb{Z}}$ be the associated helix. Then, for any $i \in \mathbb{Z}$ and any $E_{t}^{i} \in \mathcal{E}_{i}, \operatorname{Reg}_{\mathcal{B}}\left(E_{t}^{i}\right)=-i$.

Proof. First of all we will see that $\operatorname{Reg}_{\mathcal{B}}\left(E_{t}^{i}\right) \leq-i$. By the orthogonality relation (3.4), for $q>0$ and $-n \leq p \leq 0$, we have

$$
\begin{gathered}
\bigoplus_{s=1}^{\alpha_{i+p}} \operatorname{Ext}^{q}\left(R_{\mathcal{E}_{i} \cdots \mathcal{E}_{i+p+1}} E_{s}^{i+p}, E_{t}^{i}\right)=0 \quad \text { for }-n \leq p \leq-1, \quad \text { and } \\
\bigoplus_{s=1}^{\alpha_{i}} \operatorname{Ext}^{q}\left(E_{s}^{i}, E_{t}^{i}\right)=0 \quad \text { for } p=0
\end{gathered}
$$


So, $E_{t}^{i}$ is $(-i)$-regular with respect to $\mathcal{B}$ or, equivalently, $\operatorname{Reg}_{\mathcal{B}}\left(E_{t}^{i}\right) \leq-i$.

Let us now see that $E_{t}^{i}$ is not $(-i-1)$-regular with respect to $\mathcal{B}$. To this end, it is enough to see that

$$
\bigoplus_{s=1}^{\alpha_{i}} \operatorname{Ext}^{1}\left(R_{\mathcal{E}_{i+1}} E_{s}^{i}, E_{t}^{i}\right) \neq 0
$$

To prove it, we write $i=\alpha n+j$ with $0 \leq j<n, \alpha \in \mathbb{Z}$. We consider the $n$-block collection

$$
\mathcal{B}_{\alpha n}=\left(\mathcal{E}_{\alpha n}, \mathcal{E}_{\alpha n+1}, \ldots, \mathcal{E}_{\alpha n+j}=\mathcal{E}_{i}, \ldots, \mathcal{E}_{\alpha n+n}\right)
$$

and its left dual $n$-block collection

$$
\left(\mathcal{E}_{\alpha n+n}, R^{(1)} \mathcal{E}_{\alpha n+n-1}, \ldots, R^{(n-j)} \mathcal{E}_{\alpha n+j}, \ldots, R^{(n)} \mathcal{E}_{\alpha n}\right)
$$

By Remark 3.6 and the equality (3.6)

$$
\operatorname{Ext}^{n-j}\left(R^{(n-j)} E_{t}^{i}, E_{t}^{i}\right)=\operatorname{Ext}^{n-j}\left(R_{\mathcal{E}_{\alpha n+n} \cdots \mathcal{E}_{i+2} \mathcal{E}_{i+1}} E_{t}^{i}, E_{t}^{i}\right)=\mathbb{C} .
$$

So, if $j=n-1$ we are done. Assume $j<n-1$. Since $\operatorname{Ext}^{q}\left(E_{s}^{i}, E_{t}^{i}\right)=0$ for $q>0$ and $1 \leq s \leq \alpha_{i}$, applying the contravariant functor $\operatorname{Hom}\left(\cdot, E_{t}^{i}\right)$ to the exact sequence

$$
\begin{array}{r}
0 \longrightarrow R_{\mathcal{E}_{\alpha n+n-1} \cdots \mathcal{E}_{i+1}} E_{t}^{i} \longrightarrow \bigoplus_{s=1}^{\alpha_{i}} \operatorname{Hom}^{*}\left(R_{\mathcal{E}_{\alpha n+n-1} \cdots \mathcal{E}_{i+1}} E_{t}^{i}, E_{s}^{i}\right) \otimes E_{s}^{i} \\
\longrightarrow R^{(n-j)} E_{t}^{i} \longrightarrow 0
\end{array}
$$

we obtain

$$
\operatorname{Ext}^{n-j-1}\left(R_{\mathcal{E}_{\alpha n+n-1} \cdots \mathcal{E}_{i+1}} E_{t}^{i}, E_{t}^{i}\right)=\mathbb{C} .
$$

We repeat the process using the consequent right mutations and we get

$$
\operatorname{Ext}^{n-j-k}\left(R_{\mathcal{E}_{\alpha n+n-k} \cdots \mathcal{E}_{i+1}} E_{t}^{i}, E_{t}^{i}\right)=\mathbb{C}
$$

for $0 \leq k \leq n-1-j$. In particular,

$$
\operatorname{Ext}^{1}\left(R_{\mathcal{E}_{i+1}} E_{t}^{i}, E_{t}^{i}\right)=\mathbb{C}
$$

and, hence,

$$
\bigoplus_{s=1}^{\alpha_{i}} \operatorname{Ext}^{1}\left(R_{\mathcal{E}_{i+1}} E_{s}^{i}, E_{t}^{i}\right) \neq 0
$$

which implies that $E_{t}^{i}$ is not $(-i-1)$-regular and we conclude that $\operatorname{Reg}_{\mathcal{B}}\left(E_{t}^{i}\right)$ $=-i$. 
Let us now compare our new definition of regularity with the previous ones.

Castelnuovo-Mumford Regularity. In [19], Lecture 14, D. Mumford defined the notion of regularity for a coherent sheaf over a projective space. Let us recall it.

Definition 4.10. A coherent sheaf $F$ on $\mathbb{P}^{n}$ is said to be $m$-regular in the sense of Castelnuovo-Mumford if $H^{i}\left(\mathbb{P}^{n}, F(m-i)\right)=0$ for $i>0$. We define the Castelnuovo-Mumford regularity of $F, \operatorname{Reg}^{C M}(F)$, as the least integer $m$ such that $F$ is $m$-regular. We say that the Castelnuovo-Mumford regularity is $-\infty$ if such integer does not exist.

Let us now establish for coherent sheaves on $\mathbb{P}^{n}$ the agreement of the $\mathcal{B}$-regularity in the sense of Definition 4.5 with Castelnuovo-Mumford definition.

Proposition 4.11. A coherent sheaf $F$ on $\mathbb{P}^{n}$ is $m$-regular in the sense of Castelnuovo-Mumford if and only if it is $m$-regular with respect to the $n$-block collection $\mathcal{B}=\left(\mathcal{O}_{\mathbb{P}^{n}}, \mathcal{O}_{\mathbb{P}^{n}}(1), \ldots, \mathcal{O}_{\mathbb{P}^{n}}(n)\right)$ in the sense of Definition 4.5. Hence, we have

$$
\operatorname{Reg}_{\mathcal{B}}(F)=\operatorname{Reg}^{C M}(F)
$$

Proof. Since $\mathcal{B}$ is not only an $n$-block collection but also a geometric collection, the result follows from [6]; Proposition 4.6.

Chipalkatti's Regularity. In [4], Definition 1.1, J. V. Chipalkatti introduced the notion of regularity for a coherent sheaf on a Grassmannian variety and he showed that when the Grassmannian is a projective space his definition of regularity agrees with Castelnuovo-Mumford regularity. We will now see that the notion of regularity introduced in Definition 4.6 is closely related to Chipalkatti's regularity but they do not coincide. Let us recall Chipalkatti's definition.

Definition 4.12. A sheaf $F$ on $X=G r(k, n)$ is regular if $H^{q}(X, F \otimes$ $\left.\Sigma^{\beta} Q^{*}\right)=0$ for all $\beta$ such that $k \geq \beta_{1} \geq \cdots \geq \beta_{n-k} \geq 0$ and all $q \geq 1$. It is said to be $m$-regular if $F \otimes \mathcal{O}_{X}(m)$ is regular. $\operatorname{Reg}^{C}(F)$ is the least integer $m$ such that $F(m)$ is regular, set $\operatorname{Reg}^{C}(F)=-\infty$ if there is no such integer. 
Proposition 4.11 together with [4]; Theorems 1.5 and 1.6, establishes, when the Grassmannian is a projective space, the agreement of Chipalkatti's definition (Definition 4.12), Castelnuovo-Mumford's definition (Definition 4.10) and our definition (Definition 4.5). Nevertheless, next example shows that, in general, Chipalkatti's definition and our definition do not coincide.

EXAMPLE 4.13. We consider the 4-dimensional Grassmann variety $X=G r(2,4)$ and the 4-block collection described in Example 3.2 (2) tensored with $\mathcal{O}_{X}(2)$

$$
\begin{aligned}
& \mathcal{B}=\left(\mathcal{E}_{0}, \mathcal{E}_{1}, \mathcal{E}_{2}, \mathcal{E}_{3}, \mathcal{E}_{4}\right)=\left(\Sigma^{(2,2)} \mathcal{S} \otimes \mathcal{O}_{X}(2), \Sigma^{(2,1)} \mathcal{S} \otimes \mathcal{O}_{X}(2)\right. \\
& \left.\left(\Sigma^{(2,0)} \mathcal{S} \otimes \mathcal{O}_{X}(2), \Sigma^{(1,1)} \mathcal{S} \otimes \mathcal{O}_{X}(2)\right), \Sigma^{(1,0)} \mathcal{S} \otimes \mathcal{O}_{X}(2), \Sigma^{(0,0)} \mathcal{S} \otimes \mathcal{O}_{X}(2)\right)
\end{aligned}
$$

Since $\Sigma^{(2,2)} \mathcal{S} \otimes \mathcal{O}_{X}(2)=\mathcal{O}_{X}$ and $\Sigma^{(0,0)} \mathcal{S} \otimes \mathcal{O}_{X}(2)=\mathcal{O}_{X}(2)$, by [4]; Example 1.3 (a), $\operatorname{Reg}^{C}\left(\Sigma^{(0,0)} \mathcal{S} \otimes \mathcal{O}_{X}(2)\right)=-2$ and $\operatorname{Reg}^{C}\left(\Sigma^{(2,2)} \mathcal{S} \otimes \mathcal{O}_{X}(2)\right)=0$. On the other hand, by Proposition $4.9, \operatorname{Reg}_{\mathcal{B}}\left(\Sigma^{(0,0)} \mathcal{S} \otimes \mathcal{O}_{X}(2)\right)=-4$ and $\operatorname{Reg}_{\mathcal{B}}\left(\Sigma^{(2,2)} \mathcal{S} \otimes \mathcal{O}_{X}(2)\right)=0$.

Hoffman-Wang Regularity. In [14], J. W. Hoffman and H. H. Wang introduced a multigraded variant of the Castelnuovo-Mumford regularity and we will devote Section 5 of this paper to relate it to our new definition of regularity.

To emphasize the similarities between the new notion of regularity and the original definition in Chapter 14 of [19], we will end this section proving that the basic formal properties of Castelnuovo-Mumford regularity of coherent sheaves over projective spaces remain to be true in this new setting.

Proposition 4.14. Let $X$ be a smooth projective variety of dimension $n$ with an $n$-block collection of coherent sheaves $\mathcal{B}=\left(\mathcal{E}_{0}, \mathcal{E}_{1}, \ldots, \mathcal{E}_{n}\right), \mathcal{E}_{j}=$ $\left(E_{1}^{j}, \ldots, E_{\alpha_{j}}^{j}\right)$, which generates $\mathcal{D}$ and let $F$ be a coherent $\mathcal{O}_{X}$-module. If $F$ is $m$-regular with respect to $\mathcal{B}$ then the canonical map $\bigoplus_{s=1}^{\alpha_{-m}} \operatorname{Hom}\left(E_{s}^{-m}, F\right) \otimes$ $E_{s}^{-m} \rightarrow F$ is surjective and $F$ is $k$-regular with respect to $\mathcal{B}$ for any $k \geq m$ as well.

Proof. The first assertion follows from the exact sequence (4.2). To prove the second assertion it is enough to check it for $k=m+1$. Since $F$ is $m$-regular with respect to $\mathcal{B}$ we have for $q>0$

$$
\begin{cases}\bigoplus_{s=1}^{\alpha_{-m+p}} \operatorname{Ext}^{q}\left(R_{\mathcal{E}_{-m} \cdots \mathcal{E}_{-m+p+1}} E_{s}^{-m+p}, F\right)=0 & \text { if }-n \leq p \leq-1 \\ \bigoplus_{s=1}^{\alpha_{-m}} \operatorname{Ext}^{q}\left(E_{s}^{-m}, F\right)=0 & \text { if } p=0 .\end{cases}
$$


In order to see that $F$ is $(m+1)$-regular with respect to $\mathcal{B}$ we have to prove

$$
\begin{cases}\bigoplus_{s=1}^{\alpha_{-m-1+p}} \operatorname{Ext}^{q}\left(R_{\mathcal{E}_{-m-1} \cdots \mathcal{E}_{-m+p}} E_{s}^{-m-1+p}, F\right)=0 \\ \bigoplus_{s=1}^{\alpha_{-m-1}} \operatorname{Ext}^{q}\left(E_{s}^{-m-1}, F\right)=0 & \text { for } q>0,-n \leq p \leq-1 \\ & \text { for } q>0, p=0 .\end{cases}
$$

Using the equalities (4.3) and applying, for any $s, 1 \leq s \leq \alpha_{-m-1}$, the functor $\operatorname{Hom}(\cdot, F)$ to the exact sequence

$$
\begin{aligned}
0 \longrightarrow E_{s}^{-m-1} \longrightarrow \bigoplus_{t=1}^{\alpha_{-m}} \operatorname{Hom}^{*}\left(E_{s}^{-m-1}, E_{t}^{-m}\right) \otimes E_{t}^{-m} & \\
& \longrightarrow R_{\mathcal{E}_{-m}} E_{s}^{-m-1} \longrightarrow 0
\end{aligned}
$$

we obtain

$$
\operatorname{Ext}^{q}\left(E_{s}^{-m-1}, F\right)=0 \text { for } q>0 \text { and } 1 \leq s \leq \alpha_{-m-1}
$$

and, hence,

$$
\bigoplus_{s=1}^{\alpha_{-m-1}} \operatorname{Ext}^{q}\left(E_{s}^{-m-1}, F\right)=0 \quad \text { for } q>0
$$

Using again the equalities (4.3) and, for any $s, 1 \leq s \leq \alpha_{-m-2}$, the exact sequence

$$
\begin{aligned}
0 \longrightarrow R_{\mathcal{E}_{-m-1}} E_{s}^{-m-2} \longrightarrow \bigoplus_{t=1}^{\alpha_{-m}} \operatorname{Hom}^{*}\left(R_{\mathcal{E}_{-m-1}} E_{s}^{-m-2}, E_{t}^{-m}\right) \otimes E_{t}^{-m} \\
\longrightarrow R_{\mathcal{E}_{-m} \mathcal{E}_{-m-1}} E_{s}^{-m-2} \longrightarrow 0
\end{aligned}
$$

we get

$$
\bigoplus_{s=1}^{\alpha_{-m-2}} \operatorname{Ext}^{q}\left(R_{\mathcal{E}_{-m-1}} E_{s}^{-m-2}, F\right)=0 \quad \text { for any } q>0 .
$$

Going on and using the consequent right mutations of blocks, we get for all $p,-n+1 \leq p \leq-1$,

$$
\bigoplus_{s=1}^{\alpha_{-m-1+p}} \operatorname{Ext}^{q}\left(R_{\mathcal{E}_{-m-1} \cdots \mathcal{E}_{-m+p}} E_{s}^{-m-1+p}, F\right)=0 \quad \text { for all } q>0 .
$$

Therefore, it only remains to see that

$$
\bigoplus_{s=1}^{\alpha_{-m-1-n}} \operatorname{Ext}^{q}\left(R_{\mathcal{E}_{-m-1} \cdots \mathcal{E}_{-m-n}} E_{s}^{-m-1-n}, F\right)=0 \quad \text { for all } q>0
$$


The vanishing of these last Ext's groups follows again from the equalities (4.3) taking into account that, by Definition 4.1

$$
R_{\mathcal{E}_{-m-1}} R_{\mathcal{E}_{-m-2}} \cdots R_{\mathcal{E}_{-m-n}} \mathcal{E}_{-m-n-1}=R^{(n)} \mathcal{E}_{-m-n-1}=\mathcal{E}_{-m}
$$

Proposition 4.15. Let $X$ be a smooth projective variety of dimension $n$ with an $n$-block collection of coherent sheaves $\mathcal{B}=\left(\mathcal{E}_{0}, \mathcal{E}_{1}, \ldots, \mathcal{E}_{n}\right), \mathcal{E}_{j}=$ $\left(E_{1}^{j}, \ldots, E_{\alpha_{j}}^{j}\right)$, which generates $\mathcal{D}$. Let $F$ and $G$ be coherent $\mathcal{O}_{X}$-modules and let

$$
0 \longrightarrow F_{1} \longrightarrow F_{2} \longrightarrow F_{3} \longrightarrow 0
$$

be an exact sequence of coherent $\mathcal{O}_{X}$-modules. Then,

(a) $\operatorname{Reg}_{\mathcal{B}}\left(F_{2}\right) \leq \max \left\{\operatorname{Reg}_{\mathcal{B}}\left(F_{1}\right), \operatorname{Reg}_{\mathcal{B}}\left(F_{3}\right)\right\}$

(b) $\operatorname{Reg}_{\mathcal{B}}(F \oplus G)=\max \left\{\operatorname{Reg}_{\mathcal{B}}(F), \operatorname{Reg}_{\mathcal{B}}(G)\right\}$.

Proof. (a) Let $m=\max \left\{\operatorname{Reg}_{\mathcal{B}}\left(F_{1}\right), \operatorname{Reg}_{\mathcal{B}}\left(F_{3}\right)\right\}$. Since, by Proposition $4.14, F_{1}$ and $F_{3}$ are both $m$-regular with respect to $\mathcal{B}$ considering the long exact sequences

$$
\begin{aligned}
\cdots & \longrightarrow \bigoplus_{s=1}^{\alpha-m+p} \operatorname{Ext}^{q}\left(R_{\mathcal{E}_{-m} \cdots \mathcal{E}_{-m+p+1}} E_{s}^{-m+p}, F_{1}\right) \\
& \longrightarrow \bigoplus_{s=1}^{\alpha_{-m+p}} \operatorname{Ext}^{q}\left(R_{\mathcal{E}_{-m} \cdots \mathcal{E}_{-m+p+1}} E_{s}^{-m+p}, F_{2}\right) \\
& \longrightarrow \bigoplus_{s=1}^{\alpha-m+p} \operatorname{Ext}^{q}\left(R_{\mathcal{E}_{-m} \cdots \mathcal{E}_{-m+p+1}} E_{s}^{-m+p}, F_{3}\right) \longrightarrow \cdots
\end{aligned}
$$

and

$$
\begin{aligned}
\cdots & \longrightarrow \bigoplus_{s=1}^{\alpha_{-m}} \operatorname{Ext}^{q}\left(E_{s}^{-m}, F_{1}\right) \longrightarrow \bigoplus_{s=1}^{\alpha_{-m}} \operatorname{Ext}^{q}\left(E_{s}^{-m}, F_{2}\right) \\
& \longrightarrow \bigoplus_{s=1}^{\alpha_{-m}} \operatorname{Ext}^{q}\left(E_{s}^{-m}, F_{3}\right) \longrightarrow \cdots
\end{aligned}
$$

associated to (4.5) we get

$$
\begin{cases}\bigoplus_{s=1}^{\alpha-m+p} \operatorname{Ext}^{q}\left(R_{\mathcal{E}_{-m} \cdots \mathcal{E}_{-m+p+1}} E_{s}^{-m+p}, F_{2}\right)=0 & \text { if }-n \leq p \leq-1 \\ \bigoplus_{s=1}^{\alpha_{-m}} \operatorname{Ext}^{q}\left(E_{s}^{-m}, F_{2}\right)=0 & \text { if } p=0\end{cases}
$$

which implies that $\operatorname{Reg}_{\mathcal{B}}\left(F_{2}\right) \leq m$.

(b) It easily follows from the additivity of the functor

$$
\operatorname{Ext}^{q}\left(R_{\mathcal{E}_{-m} \cdots \mathcal{E}_{-m+p+1}} E_{s}^{-m+p}, \cdot\right) .
$$




\section{$\S 5$. Regularity of sheaves on multiprojective spaces}

In this section, we will restrict our attention to coherent sheaves over multiprojective spaces $X=\mathbb{P}^{n_{1}} \times \cdots \times \mathbb{P}^{n_{r}}$ and we will relate our definition of regularity to the multigraded variant of the Castelnuovo-Mumford regularity introduced by Hoffman and Wang [14] (see also [18]).

We first fix the notation we need in this section. For each integer $i>0$, let

$$
\begin{aligned}
S t_{i} & =\left\{(l, s) \in \mathbb{Z}^{2} \mid l+s=-1-i, l<0, s<0\right\} \\
& =\{(-i,-1),(-i+1,-2), \ldots,(-2,-i+1),(-1,-i)\},
\end{aligned}
$$

for $i \leq 0$, let

$$
\begin{aligned}
S t_{i} & =\left\{(l, s) \in \mathbb{Z}^{2} \mid l+s=-i, l \geq 0, s \geq 0\right\} \\
& =\{(-i, 0),(-i-1,1), \ldots,(1,-i-1),(0,-i)\} .
\end{aligned}
$$

For each $\left(p, p^{\prime}\right) \in \mathbb{Z}^{2}$, let $S t_{i}\left(p, p^{\prime}\right)=\left(p, p^{\prime}\right)+S t_{i}$.

Definition 5.1. Let $F$ be a coherent sheaf on $X=\mathbb{P}^{m} \times \mathbb{P}^{n}$. We say that $F$ is $\left(p, p^{\prime}\right)$-regular if, for all $i \geq 1$,

$$
H^{i}\left(X, F\left(k, k^{\prime}\right)\right)=0
$$

whenever $\left(k, k^{\prime}\right) \in S t_{i}\left(p, p^{\prime}\right)$.

Remark 5.2. Definition 5.1 generalizes in an obvious way to coherent sheaves on multiprojective spaces $\mathbb{P}^{n_{1}} \times \cdots \times \mathbb{P}^{n_{r}}$.

Set $d=m+n, X=\mathbb{P}^{m} \times \mathbb{P}^{n}$ and denote by $\mathcal{B}=\left(\mathcal{E}_{0}, \mathcal{E}_{1}, \ldots, \mathcal{E}_{d}\right)$ the $d$-block collection where for any $0 \leq j \leq d$, we have

$$
\mathcal{E}_{j}=\left\{\mathcal{O}_{X}(a, b) \mid a+b=j-d, 0 \geq a \geq-m, 0 \geq b \geq-n\right\}
$$

and we set $\alpha_{j}:=\sharp \mathcal{E}_{j}$. By Remark 3.6, the left dual $d$-block collection

$$
\left(R^{(0)} \mathcal{E}_{d}, R^{(1)} \mathcal{E}_{d-1}, \ldots, R^{(j)} \mathcal{E}_{d-j}, \ldots, R^{(d)} \mathcal{E}_{0}\right)
$$

of $\mathcal{B}$ is univocally determined by the orthogonality relations (3.5) and (3.6), and an intricate computation using Künneth formula for locally free sheaves on algebraic varieties shows that for any $\mathcal{O}_{X}(a, b) \in \mathcal{E}_{d-j}$ and any $0 \leq j \leq d$ we have

$$
R^{(j)} \mathcal{O}_{X}(a, b)=\bigwedge^{-a} T_{\mathbb{P}^{m}}(a) \otimes \bigwedge^{-b} T_{\mathbb{P}^{n}}(b)
$$


LEMMA 5.3. With the above notation, let $\mathcal{H}_{\mathcal{B}}=\left\{\mathcal{E}_{i}\right\}_{i \in \mathbb{Z}}$ be the helix of blocks associated to $\mathcal{B}$. Let us denote by $\mathcal{B}_{k(d+1)}$ the d-block collection of $d+1$ consecutive blocks $\left(\mathcal{E}_{k(d+1)}, \mathcal{E}_{k(d+1)+1}, \ldots, \mathcal{E}_{k(d+1)+d}\right)$. Then, we have

(1) $\mathcal{E}_{k(d+1)+i}=\left\{\mathcal{O}_{X}(a+k(m+1), b+k(n+1)) \mid a+b=i-d, 0 \geq a \geq\right.$ $-m, 0 \geq b \geq-n\}$

(2) The left dual d-block collection of $\mathcal{B}_{k(d+1)}$ is

$$
\left(R^{(0)} \mathcal{E}_{k(d+1)+d}, R^{(1)} \mathcal{E}_{k(d+1)+d-1}, \ldots, R^{(j)} \mathcal{E}_{k(d+1)+d-j}, \ldots R^{(d)} \mathcal{E}_{k(d+1)}\right)
$$

where for any $\mathcal{O}_{X}(a+k(m+1), b+k(n+1)) \in \mathcal{E}_{k(d+1)+d-j}$

$$
\begin{aligned}
R^{(j)} & \mathcal{O}_{X}(a+k(m+1), b+k(n+1)) \\
& =\bigwedge^{-a} T_{\mathbb{P}^{m}}(a+k(m+1)) \otimes \bigwedge^{-b} T_{\mathbb{P}^{n}}(b+k(n+1)) .
\end{aligned}
$$

Proof. (1) Applying Corollary 4.4, we get $\mathcal{E}_{k(d+1)+i}=\mathcal{E}_{i} \otimes K_{X}^{-k}$ and the result follows taking into account that $K_{X}^{-\lambda}=\mathcal{O}_{X}(\lambda(m+1), \lambda(n+1))$.

(2) Straightforward computation taking into account that the left dual $d$-block collection of $\mathcal{B}_{0}=\left(\mathcal{E}_{0}, \mathcal{E}_{1}, \ldots, \mathcal{E}_{d}\right)$ is determined (up to isomorphism) by (5.1), Lemma 3.12 and the equalities

$$
\begin{aligned}
R^{(j)} \mathcal{E}_{k(d+1)+d-j} & :=R_{\mathcal{E}_{k(d+1)+d} \cdots \mathcal{E}_{k(d+1)+d-j+1}} \mathcal{E}_{k(d+1)+d-j} \\
& =R_{\mathcal{E}_{d} \otimes K_{X}^{-k} \cdots \mathcal{E}_{d-j+1} \otimes K_{X}^{-k}} \mathcal{E}_{d-j} \otimes K_{X}^{-k} \\
& =R^{(j)}\left(\mathcal{E}_{d-j} \otimes K_{X}^{-k}\right) \\
& =\left(R_{\mathcal{E}_{d} \cdots \mathcal{E}_{d-j+1}} \mathcal{E}_{d-j}\right) \otimes K_{X}^{-k} \\
& =\left(R^{(j)} \mathcal{E}_{d-j}\right) \otimes K_{X}^{-k} .
\end{aligned}
$$

We have the following technical lemma.

Lemma 5.4. Let $F$ be a coherent sheaf on $X=\mathbb{P}^{m} \times \mathbb{P}^{n}$. We have:

(a) $F$ is $\left(p, p^{\prime}\right)$-regular in the sense of Hoffman and Wang if and only if $F\left(p, p^{\prime}\right)$ is $(0,0)$-regular in the sense of Hoffman and Wang.

(b) $F$ is $k(d+1)+t$-regular with respect to $\mathcal{B}$ in the sense of Definition 4.5 if and only if $F(k(m+1), k(n+1))$ is t-regular with respect to $\mathcal{B}$ in the sense of Definition 4.5 . 
Proof. (a) It obviously follows from Definition 5.1.

(b) By Definition $4.5, F$ is $k(d+1)+t$-regular with respect to $\mathcal{B}$ if and only if for $q>0$ we have

$$
\begin{cases}\bigoplus_{s=1}^{\beta_{-k(d+1)-t+p}} \operatorname{Ext}^{q}\left(R_{\mathcal{E}_{-k(d+1)-t} \cdots \mathcal{E}_{-k(d+1)-t+p+1}} E_{s}^{-k(d+1)-t+p}, F\right)=0 \\ \bigoplus_{s=1}^{\beta_{-k(d+1)-t}} \operatorname{Ext}^{q}\left(E_{s}^{-k(d+1)-t}, F\right)=0 & \text { if }-d \leq p \leq-1 \\ & \text { if } p=0 .\end{cases}
$$

Since $\mathcal{E}_{-k(d+1)+i} \cong \mathcal{E}_{i} \otimes K_{X}^{k}$, applying Lemma 3.12 , it is equivalent to say that for $q>0$, we have

$$
\begin{cases}\bigoplus_{s=1}^{\beta_{-t+p}} \operatorname{Ext}^{q}\left(R_{\mathcal{E}_{-t} \cdots \mathcal{E}_{-t+p+1}} E_{s}^{-t+p} \otimes K_{X}^{k}, F\right)=0 & \text { if }-d \leq p \leq-1 \\ \bigoplus_{s=1}^{\beta_{-t}} \operatorname{Ext}^{q}\left(E_{s}^{-t} \otimes K_{X}^{k}, F\right)=0 & \text { if } p=0\end{cases}
$$

or, equivalent, for $q>0$, we have

$$
\begin{cases}\bigoplus_{s=1}^{\beta_{-t+p}} \operatorname{Ext}^{q}\left(R_{\mathcal{E}_{-t} \cdots \mathcal{E}_{-t+p+1}} E_{s}^{-t+p}, F \otimes K_{X}^{-k}\right)=0 & \text { if }-d \leq p \leq-1 \\ \bigoplus_{s=1}^{\beta_{-t}} \operatorname{Ext}^{q}\left(E_{s}^{-t}, F \otimes K_{X}^{-k}\right)=0 & \text { if } p=0\end{cases}
$$

which means that $F(k(m+1), k(n+1))$ is $t$-regular with respect to $\mathcal{B}$ in the sense of Definition 4.5.

We are now ready to state the main result of this section.

THEOREM 5.5. Let $F$ be a coherent sheaf on $X=\mathbb{P}^{m} \times \mathbb{P}^{n}$ and set $d=n+m$. Then $F$ is (0,0)-regular in the sense of Hoffman and Wang if and only if $F$ is $(-d)$-regular with respect to $\mathcal{B}$ in the sense of Definition 4.5.

Proof. According to Definitions 5.1 and 4.5 we have to see that

$$
H^{i}(X, F(r, s))=0 \quad \text { for all } i>0, r+s=-i-1, r<0, s<0
$$

if and only if for $q>0$ we have

$$
\begin{cases}\bigoplus_{s=1}^{\alpha_{p+d}} \operatorname{Ext}^{q}\left(R_{\mathcal{E}_{d} \cdots \mathcal{E}_{p+1+d}} E_{s}^{p+d}, F\right) & \\ =\bigoplus_{\substack{a+b=p \\ 0 \geq a \geq-m \\ 0 \geq b \geq-n}} H^{q}\left(\Omega_{\mathbb{P} m}^{-a}(-a) \otimes \Omega_{\mathbb{P} n}^{-b}(-b) \otimes F\right)=0 \\ \bigoplus_{s=1}^{\alpha_{d}} \operatorname{Ext}^{q}\left(E_{s}^{d}, F\right)=H^{q}(X, F)=0 & \text { for }-d \leq p \leq-1 ;\end{cases}
$$


Let us first see that (5.2) implies (5.3). Since by [14]; Proposition 2.7, any $(0,0)$-regular sheaf is also $\left(p, p^{\prime}\right)$-regular for $p \geq 0, p^{\prime} \geq 0$, we have

$$
H^{i}(X, F(r, s))=0 \text { for all } i>0, r+s \geq-i-1, r \geq-i, s \geq-i .
$$

We will see that (5.4) implies the following stronger result

$$
H^{q}\left(\Omega_{\mathbb{P}^{m}}^{-a}(-a) \otimes \Omega_{\mathbb{P}^{n}}^{-b}(-b) \otimes F(r, s)\right)=0
$$

for any $q>0,0 \geq a \geq-m, 0 \geq b \geq-n, r+s \geq-q+1$ and $r, s \geq-q+1$. To this end, we will first prove the following claim:

Claim. For any $i>0,0 \geq b \geq-n, r+s \geq-i, r \geq-i$ and $s \geq-i+1$

$$
H^{i}\left(\Omega_{\mathbb{P}^{n}}^{-b}(-b) \otimes F(r, s)\right)=0 .
$$

Proof of Claim. We will prove it by induction on $b$. By (5.4), for $i>0$, $b=-n, r+s \geq-i, r \geq-i$ and $s \geq-i+1$, we have

$$
H^{i}\left(\Omega_{\mathbb{P}^{n}}^{n}(n) \otimes F(r, s)\right)=H^{i}(F(r, s-1))=0 .
$$

Now take $0 \leq b<-n$ and consider on $X$ the exact sequence

$$
\begin{aligned}
0 \longrightarrow \Omega_{\mathbb{P}^{n}}^{-b+1}(-b+1) \otimes F(r, s-1) \longrightarrow \mathcal{O}_{\mathbb{P}^{n}}(-1)^{\left(\begin{array}{c}
n+1 \\
-b+1
\end{array}\right) \otimes F(r, s)} & \longrightarrow \Omega_{\mathbb{P}^{n}}^{-b}(-b) \otimes F(r, s) \longrightarrow 0
\end{aligned}
$$

and the cohomological exact sequence associated to it

$$
\begin{aligned}
& \cdots \longrightarrow H^{i}\left(F(r, s-1)^{\left(\begin{array}{c}
n+1 \\
-b+1
\end{array}\right)}\right) \longrightarrow H^{i}\left(\Omega_{\mathbb{P}^{n}}^{-b}(-b) \otimes F(r, s)\right) \\
& \longrightarrow H^{i+1}\left(\Omega_{\mathbb{P}^{n}}^{-b+1}(-b+1) \otimes F(r, s-1)\right) \longrightarrow \cdots .
\end{aligned}
$$

Applying (5.4) and hypothesis of induction we get $H^{i}\left(\Omega_{\mathbb{P} n}^{-b}(-b) \otimes F(r, s)\right)=0$ for all $i>0, r+s \geq-i, r \geq-i$ and $s \geq-i+1$ which finishes the proof of the claim.

Let us now prove (5.5) by decreasing induction on $p:=-a-b, 0 \leq p \leq$ $d$. If $p=d$, then $a=-m, b=-n$ and by (5.4) for any $q>0, r+s \geq-q+1$ and $r, s \geq-q+1$ we have

$$
H^{q}\left(\Omega_{\mathbb{P}^{m}}^{m}(m) \otimes \Omega_{\mathbb{P}^{n}}^{n}(n) \otimes F(r, s)\right)=H^{q}(F(r-1, s-1))=0 .
$$


Assume that (5.5) holds for $p+1$ and fix $a, b$ such that $0<p=-a-b<d$. If $a=0$ or $a=-m$ the result follows from the claim. So, we can assume $0>a>-m$ and we consider on $X$ the exact sequence

$$
\begin{aligned}
& 0 \longrightarrow \Omega_{\mathbb{P}^{m}}^{-a+1}(-a+1) \otimes \Omega_{\mathbb{P}^{n}}^{-b}(-b) \otimes F(r-1, s) \\
& \longrightarrow \mathcal{O}_{\mathbb{P} m}(-1)^{\left(\begin{array}{c}
m+1 \\
-a+1
\end{array}\right)} \otimes \Omega_{\mathbb{P} n}^{-b}(-b) \otimes F(r, s) \\
& \longrightarrow \Omega_{\mathbb{P}^{m}}^{-a}(-a) \otimes \Omega_{\mathbb{P}^{n}}^{-b}(-b) \otimes F(r, s) \longrightarrow 0
\end{aligned}
$$

and the cohomological exact sequence associated to it

$$
\begin{aligned}
\cdots & \longrightarrow H^{q}\left(\mathcal{O}_{\mathbb{P}^{m}}(-1)^{\left(\begin{array}{c}
m+1 \\
-a+1
\end{array}\right)} \otimes \Omega_{\mathbb{P}^{n}}^{-b}(-b) \otimes F(r, s)\right) \\
& \longrightarrow H^{q}\left(\Omega_{\mathbb{P}^{m}}^{-a}(-a) \otimes \Omega_{\mathbb{P}^{n}}^{-b}(-b) \otimes F(r, s)\right) \\
& \longrightarrow H^{q+1}\left(\Omega_{\mathbb{P}^{m}}^{-a+1}(-a+1) \otimes \Omega_{\mathbb{P}^{n}}^{-b}(-b) \otimes F(r-1, s)\right) \longrightarrow \cdots
\end{aligned}
$$

By hypothesis of induction $H^{q+1}\left(\Omega_{\mathbb{P} m}^{-a+1}(-a+1) \otimes \Omega_{\mathbb{P} n}^{-b}(-b) \otimes F(r-1, s)\right)=0$ for any $q>0,0 \geq a \geq-m, 0 \geq b \geq-n, r+s \geq-q+1$ and $r, s \geq-q+1$ and it follows from the Claim that $H^{q}\left(\mathcal{O}_{\mathbb{P}^{m}}(-1)^{\left(\begin{array}{c}m+1 \\ -a+1\end{array}\right)} \otimes \Omega_{\mathbb{P}^{n}}^{-b}(-b) \otimes F(r, s)\right)=0$ for any $q>0,0 \geq a \geq-m, 0 \geq b \geq-n, r+s \geq-q+1$ and $r, s \geq-q+1$. Hence $H^{q}\left(\Omega_{\mathbb{P}^{m}}^{-a}(-a) \otimes \Omega_{\mathbb{P}^{n}}^{-b}(-b) \otimes F(r, s)\right)=0$ for any $q>0,0 \geq a \geq-m$, $0 \geq b \geq-n, r+s \geq-q+1$ and $r, s \geq-q+1$ and this finishes the proof of $(5.5)$.

Let us prove the converse. We will prove that (5.3) implies

$$
H^{i}(X, F(-s,-t))=0 \text { for all } i>0, i \geq s+t-1, s, t>0 .
$$

First of all, we will prove by induction on $t$ that for all $i \geq t$

$$
H^{i}(X, F(-1,-t))=0 .
$$

By (5.3), for $t=1$ and $i>0$ we have

$$
H^{i}(X, F(-1,-1))=H^{i}\left(X, \Omega_{\mathbb{P} m}^{m}(m) \otimes \Omega_{\mathbb{P} n}^{n}(n) \otimes F\right)=0 .
$$

For $t>1$, we consider on $X$ the exact sequence

$0 \longrightarrow F(-1,-t) \longrightarrow F(-1,-(t-1))^{n+1} \longrightarrow \Omega_{\mathbb{P} n}^{n-1}(n-t+1) \otimes F(-1,0) \longrightarrow 0$

and the cohomological exact sequence associated to it

$$
\begin{aligned}
\cdots \longrightarrow H^{i-1}\left(\Omega_{\mathbb{P} n}^{n-1}(n-t+1) \otimes F(-1,0)\right) & \longrightarrow H^{i}(F(-1,-t)) \\
& \longrightarrow H^{i}\left(F(-1,-(t-1))^{n+1}\right) \longrightarrow \cdots .
\end{aligned}
$$

By hypothesis of induction $H^{i}(F(-1,-(t-1)))=0$ for any $i>t-1$. Hence it is enough to prove the following Claim: 
Claim 1. For any $j, 0 \leq j \leq t-2$ and $i \geq j+1$,

$$
H^{i}\left(\Omega_{\mathbb{P}^{n}}^{n-t+1+j}(n-t+1) \otimes F(-1,0)\right)=0 .
$$

Proof of Claim 1. We will proceed by induction on $j$. For $j=0$ and $i>0$, by $(5.3)$

$H^{i}\left(\Omega_{\mathbb{P} n}^{n-t+1}(n-t+1) \otimes F(-1,0)\right)=H^{i}\left(\Omega_{\mathbb{P} m}^{m}(m) \otimes \Omega_{\mathbb{P} n}^{n-t+1}(n-t+1) \otimes F\right)=0$.

For $0<j \leq t-2$, consider the exact sequence on $X$

$$
\begin{aligned}
0 \longrightarrow \Omega_{\mathbb{P}^{n}}^{n-t+1+j}(n-t+1) \otimes F & (-1,0) \longrightarrow F(-1,-j)^{\left(\begin{array}{c}
n+1 \\
n+1-t+j
\end{array}\right)} \\
& \longrightarrow \Omega_{\mathbb{P}^{n}}^{n-t+j}(n-t+1) \otimes F(-1,0) \longrightarrow 0
\end{aligned}
$$

and the cohomological exact sequence associated to it

$$
\begin{gathered}
\cdots \longrightarrow H^{i-1}\left(\Omega_{\mathbb{P}^{n}}^{n-t+j}(n-t+1) \otimes F(-1,0)\right) \\
\longrightarrow H^{i}\left(\Omega_{\mathbb{P}^{n}}^{n-t+1+j}(n-t+1) \otimes F(-1,0)\right) \\
\longrightarrow H^{i}(F(-1,-j))\left(\begin{array}{c}
n+1 \\
n+1-t+j
\end{array}\right) \longrightarrow \cdots
\end{gathered}
$$

By hypothesis of induction $H^{i-1}\left(\Omega_{\mathbb{P} n}^{n-t+j}(n-t+1) \otimes F(-1,0)\right)=0$ for any $i>j$ and since $j<t$, by hypothesis of induction on $t, H^{i}(F(-1,-j))=0$ for any $i \geq j$. Thus $H^{i}\left(\Omega_{\mathbb{P} n}^{n-t+1+j}(n-t+1) \otimes F(-1,0)\right)=0$ for any $i \geq j+1$. This finishes the proof of the Claim 1 and the proof of (5.7).

By symmetry, for all $i \geq t$ we also have

$$
H^{i}(X, F(-t,-1))=0 .
$$

Now, by induction on $s>0$ we will prove that (5.6) holds for any $t>0$, $i \geq t+s-1$. The case $s=1$ is already done. Take $s>1$ and we will see that

$$
H^{i}(F(-s,-t))=0 \text { for any } t \leq i, i \geq s+t-1
$$

To this end, we will prove that the following cohomology groups vanish on $X$ :

(a) For any $i \geq s \geq 1$,

$$
H^{i}\left(\Omega_{\mathbb{P}^{n}}^{n-t+1}(n-t+1) \otimes F(-s, 0)\right)=0 .
$$


(b) For any $j, 1 \leq j \leq t-1, t>0$ and $i \geq s+t-1$,

$$
H^{i}\left(\Omega_{\mathbb{P}^{n}}^{n-t+j}(n-t+1) \otimes F(-s, 0)\right)=0 .
$$

(a) We proceed by induction on $s$. For $s=1$, by (5.3), for any $i>0$ $H^{i}\left(\Omega_{\mathbb{P}^{n}}^{n-t+1}(n-t+1) \otimes F(-1,0)\right)=H^{i}\left(\Omega_{\mathbb{P}^{m}}^{m}(m) \otimes \Omega_{\mathbb{P}^{n}}^{n-t+1}(n-t+1) \otimes F\right)=0$.

Fix $s>1$ and let us prove

Claim 2. For any $j, 1 \leq j \leq s-1$ and $i \geq j$,

$$
H^{i}\left(\Omega_{\mathbb{P}^{m}}^{m-s+j}(m-s+1) \otimes \Omega_{\mathbb{P}^{n}}^{n-t+1}(n-t+1) \otimes F\right)=0 .
$$

Proof of Claim 2. For $j=1$, by (5.3)

$$
H^{i}\left(\Omega_{\mathbb{P}^{m}}^{m-s+1}(m-s+1) \otimes \Omega_{\mathbb{P}^{n}}^{n-t+1}(n-t+1) \otimes F\right)=0 .
$$

For $1<j \leq s-1$ consider the exact sequence on $X$

$$
\begin{aligned}
0 & \longrightarrow \Omega_{\mathbb{P} m}^{m-s+j}(m-s+1) \otimes \Omega_{\mathbb{P}^{n}}^{n-t+1}(n-t+1) \otimes F \\
& \longrightarrow \Omega_{\mathbb{P}^{n}}^{n-t+1}(n-t+1) \otimes F(-j+1,0)^{\left(\begin{array}{c}
m+1 \\
m-s+j
\end{array}\right)} \\
& \longrightarrow \Omega_{\mathbb{P}^{m}}^{m-s+j-1}(m-s+1) \otimes \Omega_{\mathbb{P}^{n}}^{n-t+1}(n-t+1) \otimes F \longrightarrow 0
\end{aligned}
$$

and the cohomological exact sequence associated to it

$$
\begin{aligned}
\cdots & \longrightarrow H^{i-1}\left(\Omega_{\mathbb{P}^{m}}^{m-s+j-1}(m-s+1) \otimes \Omega_{\mathbb{P}^{n}}^{n-t+1}(n-t+1) \otimes F\right) \\
& \longrightarrow H^{i}\left(\Omega_{\mathbb{P}^{m}}^{m-s+j}(m-s+1) \otimes \Omega_{\mathbb{P}^{n}}^{n-t+1}(n-t+1) \otimes F\right) \\
& \longrightarrow H^{i}\left(\Omega_{\mathbb{P}^{n}}^{n-t+1}(n-t+1) \otimes F(-j+1,0)\right)^{\left(\begin{array}{c}
m+1 \\
m-s+j
\end{array}\right)} \longrightarrow \cdots
\end{aligned}
$$

By hypothesis of induction on $j$,

$$
H^{i-1}\left(\Omega_{\mathbb{P}^{m}}^{m-s+j-1}(m-s+1) \otimes \Omega_{\mathbb{P}^{n}}^{n-t+1}(n-t+1) \otimes F\right)=0
$$

and by hypothesis of induction on $s$, for $j-1<s$,

$$
H^{i}\left(\Omega_{\mathbb{P}^{n}}^{n-t+1}(n-t+1) \otimes F(-j+1,0)\right)=0 .
$$

Thus

$$
H^{i}\left(\Omega_{\mathbb{P}^{m}}^{m-s+j}(m-s+1) \otimes \Omega_{\mathbb{P}^{n}}^{n-t+1}(n-t+1) \otimes F\right)=0
$$

and this finishes the proof of Claim 2. 
Using the cohomological exact sequence on $X$

$$
\begin{aligned}
\cdots & \longrightarrow H^{i-1}\left(\Omega_{\mathbb{P}^{m}}^{m-1}(m-s+1) \otimes \Omega_{\mathbb{P}^{n}}^{n-t+1}(n-t+1) \otimes F\right) \\
& \longrightarrow H^{i}\left(\Omega_{\mathbb{P}^{n}}^{n-t+1}(n-t+1) \otimes F(-s, 0)\right) \\
& \longrightarrow H^{i}\left(\Omega_{\mathbb{P}^{n}}^{n-t+1}(n-t+1) \otimes F(-s+1,0)\right)^{m+1} \longrightarrow \cdots
\end{aligned}
$$

by hypothesis of induction and by Claim 2, we get that for any $i \geq s, s \geq 1$, $H^{i}\left(\Omega_{\mathbb{P n}}^{n-t+1}(n-t+1) \otimes F(-s, 0)\right)=0$ which finishes the proof of $(a)$.

(b) The case $j=1$ follows from $(a)$. Fix $j>0$ and consider the cohomological exact sequence

$$
\begin{gathered}
\cdots \longrightarrow H^{i-1}\left(\Omega_{\mathbb{P}^{n}}^{n-t+j-1}(n-t+1) \otimes F(-s, 0)\right) \\
\longrightarrow H^{i}\left(\Omega_{\mathbb{P}^{n}}^{n-t+j}(n-t+1) \otimes F(-s, 0)\right) \\
\longrightarrow H^{i}(F(-s,-j+1))^{\left(\begin{array}{c}
n+1 \\
n-t+j
\end{array}\right)} \longrightarrow \cdots
\end{gathered}
$$

associated to the exact sequence on $X$

$$
\begin{aligned}
0 \longrightarrow \Omega_{\mathbb{P}^{n}}^{n-t+j}(n-t+1) \otimes F & (-s, 0) \longrightarrow F(-s,-j+1)^{\left(\begin{array}{c}
n+1 \\
n-t+j
\end{array}\right)} \\
& \longrightarrow \Omega_{\mathbb{P}^{n-t+j-1}}(n-t+1) \otimes F(-s, 0) \longrightarrow 0 .
\end{aligned}
$$

Since $j-1<t$, by the first cases $H^{i}(F(-s,-j+1))=0$ and by hypothesis of induction $H^{i-1}\left(\Omega_{\mathbb{P} n}^{n-t+j-1}(n-t+1) \otimes F(-s, 0)\right)=0$. Thus, for any $j$, $1 \leq j \leq t-1$ and $i \geq s+t-1, H^{i}\left(\Omega_{\mathbb{P} n}^{n-t+j}(n-t+1) \otimes F(-s, 0)\right)=0$ which finishes the proof of $(b)$.

Finally, to prove (5.9), we proceed by induction on $t>0$. By (5.8), the case $t=1$ is already done, so we fix $t>1$ and we consider the following exact sequence on $X$

$0 \longrightarrow F(-s,-t) \longrightarrow F(-s,-t+1)^{n+1} \longrightarrow \Omega_{\mathbb{P} n}^{n-1}(n-t+1) \otimes F(-s, 0) \longrightarrow 0$

and the associated cohomological exact sequence

$$
\begin{aligned}
\cdots \longrightarrow H^{i-1}\left(\Omega_{\mathbb{P}^{n}}^{n-1}(n-t+1) \otimes F(-s, 0)\right) & \longrightarrow H^{i}(F(-s,-t)) \\
& \longrightarrow H^{i}(F(-s,-t+1))^{n+1} \longrightarrow \cdots .
\end{aligned}
$$

By hypothesis of induction $H^{i}(F(-s,-t+1))=0$ and it follows from $(b)$ that $H^{i-1}\left(\Omega_{\mathbb{P} n}^{n-1}(n-t+1) \otimes F(-s, 0)\right)=0$. Hence, $H^{i}(F(-s,-t))=0$ for any $t>0, i \geq s+t-1$ and this proves what we want. 
As a consequence of this last Theorem we have,

Corollary 5.6. Let $F$ be a coherent sheaf on $X=\mathbb{P}^{m} \times \mathbb{P}^{n}$. Set $d=n+m$.

(1) If $F$ is p-regular with respect to $\mathcal{B}$ in the sense of Definition 4.5 and $p=\lambda(d+1)+\rho, 0<\rho \leq d+1$, then $F$ is $((\lambda+2)(m+1),(\lambda+2)(n+1))$ regular in the sense of Hoffman and Wang; and

(2) If $F$ is $(s, r)$-regular in the sense of Hoffman and Wang with $r=$ $\lambda(m+1)+t, 0<t \leq m+1$, and $s=\mu(m+1)+x, 0<x \leq n+1$ then $F$ is $(\max (\lambda, \mu)(d+1)+1)$-regular with respect to $\mathcal{B}$ in the sense of Definition 4.5 .

Proof. (1) By Proposition 4.14, if $F$ is $p=\lambda(d+1)+\rho$-regular with respect to $\mathcal{B}$, then $F$ is $((\lambda+2)(d+1)-d)$-regular with respect to $\mathcal{B}$ as well. By Lemma 5.4, $F((\lambda+2)(m+1),(\lambda+2)(n+1))$ is $(-d)$-regular with respect to $\mathcal{B}$. Applying Theorem 5.5, we get that $F((\lambda+2)(m+1),(\lambda+$ $2)(n+1))$ is $(0,0)$-regular in the sense of Hoffman and Wang and hence $F$ is $((\lambda+2)(m+1),(\lambda+2)(n+1))$-regular in the sense of Hoffman and Wang.

(2) Set $\phi=\max (\lambda, \mu)$. By [14]; Proposition 2.7, $F$ is $((\phi+1)(m+$ $1),(\phi+1)(n+1))$-regular in the sense of Hoffman and Wang. Therefore, $F((\phi+1)(m+1),(\phi+1)(n+1))$ is $(0,0)$-regular in the sense of Hoffman and Wang and applying Theorem 5.5 we obtain that $F((\phi+1)(m+1),(\phi+$ $1)(n+1))$ is $(-d)$-regular with respect to $\mathcal{B}$ and so $F$ is $(\phi(d+1)+1)$-regular with respect to $\mathcal{B}$ in the sense of Definition 4.5.

\section{§6. Final remark and open problem}

The notion of regularity that we have introduced in Section 4 applies to any coherent sheaf on a large class of smooth projective varieties: projective spaces, multiprojective spaces, hyperquadric varieties, Grassmannians, etc. More precisely, it applies to coherent sheaves on any $n$-dimensional smooth projective variety which has an $n$-block collection $\mathcal{B}=\left(\mathcal{E}_{0}, \mathcal{E}_{1}, \ldots, \mathcal{E}_{n}\right)$ of type $\left(\alpha_{0}, \alpha_{1}, \ldots, \alpha_{n}\right)$ of coherent sheaves on $X$ which generates the derived category of bounded complexes $\mathcal{D}=D^{b}\left(\mathcal{O}_{X}-\bmod \right)$. Hence, we are led to pose the following question/problem:

Problem 6.1. To characterize $n$-dimensional smooth projective varieties which have an $n$-block collection $\mathcal{B}=\left(\mathcal{E}_{0}, \mathcal{E}_{1}, \ldots, \mathcal{E}_{n}\right), \mathcal{E}_{j}=\left(E_{1}^{j}, E_{2}^{j}, \ldots\right.$, $E_{\alpha_{j}}^{j}$ ) of coherent sheaves on $X$ which generates $\mathcal{D}$. 
Note ADded In Proof. Problem 2.10 is closely related to Dubrovin's conjecture concerning the semisimplicity of the quantum cohomology algebra. More precisely, it states

Conjecture 6.2. (Dubrovin [9]; Conjecture 4.2.2 (1)) Let $X$ be a smooth complex compact variety. The even quantum cohomology ring of $X$ is generically semisimple if and only if $X$ is a Fano variety and the category $\mathcal{D}$ admits a full exceptional collection of length equal to $\sum_{q} H^{q, q}(X)$.

\section{REFERENCES}

[1] A. A. Beilinson, Coherent sheaves on $\mathbb{P}^{n}$ and Problems of Linear Algebra, Funkt. Anal. Appl., 12 (1979), 214-216.

[2] A. I. Bondal, Representation of associative algebras and coherent sheaves, Math. USSR Izvestiya, 34 (1990), 23-42.

[3] A. I. Bondal and A. E. Polishchuk, Homological properties of associative algebras: the method of helices, Russian Acad. Sci. Izv. Math., 42 (1994), 219-259.

[4] J. V. Chipalkatti, A generalization of Castelnuovo regularity to Grassmann varieties, manusc. math., 102 (2000), 447-464.

[5] L. Costa and R. M. Miró-Roig, Tilting sheaves on toric varieties, Math. Z., 248 (2004), 849-865.

[6] L. Costa and R. M. Miró-Roig, Geometric collections and Castelnuovo-Mumford regularity, Math. Proc. Cambridge, to appear.

[7] L. Costa and R. M. Miró-Roig, Cohomological characterization of vector bundles on multiprojective spaces, J. of Algebra, 294 (2005), 73-96.

[8] J. M. Drezet and J. Le Potier, Fibrés stables et fibrés exceptionelles sur $\mathbb{P}^{2}$, Ann. Ec. Norm. Sup., 18 (1985), 193-244.

[9] B. Dubrovin, Geometric and analytic theory of Frobenius manifolds, Documenta Math. Special Issue ICM Berlin 1998, pp. 315-326.

[10] W. Fulton and J. Harris, Representation Theory: A first course, Graduate text in Math. 129, Springer-Verlag, 1991.

[11] A. L. Gorodentsev and S. A. Kuleshov, Helix Theory, Preprint MPI (2001), 97.

[12] L. Gorodentsev and A. N. Rudakov, Exceptional Vector Bundles on the Projective Space, Duke Math. J., 54 (1987), 115-130.

[13] L. Hille, Consistent algebras and special tilting sequences, Math. Z., 220 (1995), 189-205.

[14] J. W. Hoffman and H. H. Wang, Castelnuovo-Mumford regularity in biprojective spaces, Adv. Geom., 4 (2004), 513-536.

[15] M. M. Kapranov, On the derived category of coherent sheaves on Grassmann manifolds, Math. USSR Izvestiya, 24 (1985), 183-192.

[16] M. M. Kapranov, On the derived category of coherent sheaves on some homogeneous spaces, Invent. Math., 92 (1988), 479-508. 
[17] B. V. Karpov and D. Yu Nogin, Three-block exceptional collections over Del Pezzo surfaces, Math. USSR Izvestiya, 62 (1998), 429-463.

[18] D. Maclagan and G. G. Smith, Multigraded Castelnuovo-Mumford regularity, J. Reine Angew. Math., 571 (2004), 179-212.

[19] D. Mumford, Lectures on curves on an algebraic surface, Princeton University Press, Princeton, N.J., 1966.

[20] J. L. Verdier, Des catègories dèrivées des catègories abéliennes, Astérisque, 239 (1996).

L. Costa

Facultat de Matemàtiques

Departament d'Algebra i Geometria

Gran Via de les Corts Catalanes 585

08007 Barcelona

Spain

costa@ub.edu

R. M. Miró-Roig

Facultat de Matemàtiques

Departament d'Algebra i Geometria

Gran Via de les Corts Catalanes 585

08007 Barcelona

Spain

miro@ub.edu 